\title{
LOCAL LYMAN BREAK GALAXY ANALOGS: THE IMPACT OF MASSIVE STAR-FORMING CLUMPS ON THE INTERSTELLAR MEDIUM AND THE GLOBAL STRUCTURE OF YOUNG, FORMING GALAXIES
}

\author{
Roderik A. Overzier ${ }^{1}$, Timothy M. Heckman ${ }^{2}$, Christy Tremonti ${ }^{3}$, Lee Armus ${ }^{4}$, Antara Basu-Zych ${ }^{5}$, \\ Thiago Gonçalves ${ }^{6}$, R. Michael Rich ${ }^{7}$, D. Christopher Martin ${ }^{6}$, Andy PtaK $^{2}$, David Schiminovich ${ }^{8}$, Hollland C. Ford $^{2}$, \\ BARry Madore ${ }^{9}$, AND Mark SeIbert ${ }^{9}$ \\ ${ }^{1}$ Max-Planck-Institut for Astrophysics, D-85748 Garching, Germany; overzier@ mpa-garching.mpg.de \\ ${ }^{2}$ Department of Physics and Astronomy, The Johns Hopkins University, 3400 North Charles Street, Baltimore, MD 21218, USA \\ ${ }^{3}$ Max-Planck Institute for Astronomy, Königstuhl 17, D-69117, Heidelberg, Germany \\ ${ }^{4}$ Spitzer Science Center, California Institute of Technology, Pasadena, CA, USA \\ ${ }^{5}$ NASA Goddard Space Flight Center, Laboratory for X-ray Astrophysics, Greenbelt, MD 20771, USA \\ ${ }^{6}$ California Institute of Technology, MC 405-47, 1200 East California Boulevard, Pasadena, CA 91125, USA \\ ${ }^{7}$ Deptartment of Physics and Astronomy, Division of Astronomy and Astrophysics, University of California, Los Angeles, CA 90095-1562, USA \\ ${ }^{8}$ Department of Astronomy, Columbia University, MC 2457, 550 West 120th Street, New York, NY 10027, USA \\ ${ }^{9}$ Observatories of the Carnegie Institution of Washington, 813 Santa Barbara Street, Pasadena, CA 91101, USA \\ Received 2009 June 18; accepted 2009 October 7; published 2009 October 28
}

\begin{abstract}
We report on the results of Hubble Space Telescope optical and UV imaging, Spitzer mid-IR photometry, and optical spectroscopy of a sample of 30 low-redshift $(z \sim 0.1$ to 0.3$)$ galaxies chosen from the Sloan Digital Sky Survey and Galaxy Evolution Explorer surveys to be accurate local analogs of the high-redshift Lyman break galaxies. The Lyman break analogs (LBAs) are similar in stellar mass, metallicity, dust extinction, star formation rate (SFR), physical size, and gas velocity dispersion, thus enabling a detailed investigation of many processes that are important in star-forming galaxies at high redshift. The main optical emission-line properties of LBAs, including evidence for outflows, are also similar to those typically found at high redshift. This indicates that the conditions in their interstellar medium are comparable. In the UV, LBAs are characterized by complexes of massive clumps of star formation, while in the optical they most often show evidence for (post-)mergers and interactions. In six cases, we find a single extremely massive (up to several $\times 10^{9} M_{\odot}$ ) compact (radius $\sim 10^{2} \mathrm{pc}$ ) dominant central object (DCO). The DCOs are preferentially found in LBAs with the highest mid-IR luminosities $\left(L_{24 \mu \mathrm{m}}=10^{10.3}-10^{11.2} L_{\odot}\right)$ and correspondingly high SFRs $\left(15-100 M_{\odot} \mathrm{yr}^{-1}\right)$. We show that the massive starforming clumps (including the DCOs) have masses much larger than the nuclear super star clusters seen in normal late-type galaxies. However, the DCOs do have masses, sizes, and densities similar to the excess light/central cusps seen in typical elliptical galaxies with masses similar to the LBA galaxies. We suggest that the DCOs form in the present-day examples of the dissipative mergers at high redshift that are believed to have produced the central cusps in local ellipticals (consistent with the disturbed optical morphologies of the LBAs). More generally, the properties of the LBAs are consistent with the idea that instabilities in a gas-rich disk lead to very massive star-forming clumps that eventually coalesce to form a spheroid. Finally, we comment on the apparent lack of energetically significant active galactic nuclei in the DCOs. We speculate that the DCOs are too young at present to grow a supermassive black hole because they are still in a supernova-dominated outflow phase (age less than $50 \mathrm{Myr}$ ).
\end{abstract}

Key words: galaxies: active - galaxies: bulges - galaxies: high-redshift - galaxies: peculiar - galaxies: starburst

Online-only material: color figures

\section{INTRODUCTION}

One of the most remarkable features of high-redshift $(z \sim$ 2-6) galaxies is their great potential for vigorous star formation. Moreover, observations with the Hubble Space Telescope (HST) indicate that that star formation largely occurs in an extremely "clumpy" and compact mode (e.g., Cowie et al. 1995; Franx et al. 1997; Elmegreen et al. 2005; Stark et al. 2008). These clumps are reminiscent of star-forming complexes in local $\mathrm{H}$ II regions, but their total masses and scale sizes are several orders of magnitudes larger. As shown by Elmegreen et al. (2009), clumps with masses of $10^{7-9} M_{\odot}$ and sizes of $\gtrsim 1 \mathrm{kpc}$ are a very typical constituent of extended, irregular galaxies at $z=1-4$ in the Ultra Deep Field (UDF; Beckwith et al. 2006). The relatively high gas velocity dispersions observed in some high-redshift galaxies (e.g., Law et al. 2007, 2009; Förster Schreiber et al. 2009) coupled with their large inferred gas column densities and gas fractions can explain the formation of the clumps and their bulk properties from gravitational instabilities in gas-rich, Jeansunstable disks. This process may be instigated, for example, by the continuous accretion of gas or by the delivery of gasrich material in small merger events (Bournaud \& Elmegreen 2009; Elmegreen et al. 2009). In any case, the compactness of the stellar clumps furthermore implies that they can form relatively unhampered by velocity shear or supernova feedback. Simulations show that for some galaxies the coalescence of clump systems may lie at the root of the formation of galaxy bulges, and possibly even their supermassive nuclear black holes if it is assumed that each clump gave rise to its own intermediatemass black hole prior to their migration to the center (Noguchi 1999; Immeli et al. 2004; Elmegreen et al. 2008a, 2008b).

It would be extremely valuable to study similar objects in nearby galaxies where multi-waveband data with relatively high spatial resolution and sensitivity would allow the relevant physical processes to be probed in much greater detail. While local starburst galaxies used in previous studies have some 
similarities to star-forming galaxies at high redshift, there are some important differences: local starbursts having star formation rates (SFRs) of $\gtrsim 100 M_{\odot} \mathrm{yr}^{-1}$ are very dusty systems (the so-called Ultra-Luminous Infrared Galaxies, ULIRGs). The intense star formation occurs in compact, sub-kpc scale regions in the centers of interacting galaxies, and are usually inconspicuous in the far-UV (Heckman et al. 1998; Goldader et al. 2002). Although very dusty starbursts clearly make up a non-negligible fraction of high-redshift galaxies (e.g., Hughes et al. 1998; Huang et al. 2005; Papovich et al. 2006; Caputi et al. 2007; Burgarella et al. 2009; Daddi et al. 2009), most of the systems contributing to the cosmic SFR at high redshift are accounted for in deep UV-selected samples of Lyman break galaxies (LBGs; e.g., Reddy et al. 2006; Reddy \& Steidel 2009; Bouwens et al. 2009), indicating that the amount of dust extinction in LBGs is typically much lower: the average ratio of their bolometric IR-to-UV luminosities, $L_{\mathrm{IR}} / L_{\mathrm{UV}}$, is much lower $(\sim 10 \times)$ compared to local galaxies of the same $L_{\text {bol }}$ (e.g., Buat et al. 2007; Reddy et al. 2006; Burgarella et al. 2007). Locally calibrated dust corrections that are based on the reddening of the UV continuum slope as derived by Meurer et al. (1999) seem to apply to typical LBGs (Reddy et al. 2006) with some exceptions (e.g., Baker et al. 2001; Reddy et al. 2006; Siana et al. 2008), and can be used to show that, despite their UV selection, LBGs at $z \simeq 2-4$ still put out most of their energy in the mid- and far-IR (Bouwens et al. 2009).

The star formation in LBGs typically occurs over the entire extent of the galaxy ranging from a few hundred pc to several kpc (e.g., Franx et al. 1997; Giavalisco 2002; Bouwens et al. 2004; Ferguson et al. 2004; Stark et al. 2008). The relatively low extinctions and high UV surface brightnesses seen in LBGs are characteristic of local blue compact dwarf galaxies (BCDs). However, typical BCDs have SFRs up to two orders of magnitude smaller than LBGs and occur in galaxies with much lower mass (e.g., Telles \& Terlevich 1997; Hopkins et al. 2002), although some may resemble fainter LBGs at high redshift in their basic properties (see, e.g., Östlin et al. 2009). The main differences between LBGs and typical local starbursts studied previously may be due to a systematically lower metallicity and higher gas-mass fraction in the LBGs and/or due to a different triggering mechanism (e.g., Erb et al. 2006a, 2006b; Law et al. 2009).

The "Lyman break analogs" (LBA) project was designed in order to search for local starburst galaxies that share typical characteristics of high-redshift LBGs (Heckman et al. 2005). The LBA sample and main properties are given in Heckman et al. (2005), Hoopes et al. (2007), Basu-Zych et al. (2007, 2009), and Overzier et al. (2008, Paper I), and we note that the LBAs are identical to the sample of "supercompact UVluminous galaxies" (ScUVLGs) referred to in the papers listed above. In brief, the UV imaging survey performed by the Galaxy Evolution Explorer (GALEX) was used in order to select the most luminous $\left(L_{\mathrm{FUV}}>10^{10.3} L_{\odot}\right)$ and most compact $\left(I_{\mathrm{FUV}}>\right.$ $10^{9} L_{\odot} \mathrm{kpc}^{-2}$ ) star-forming galaxies at $z<0.3$. Galaxies having these high $L_{\mathrm{FUV}}$ are already extremely rare (local space density of $\sim 10^{-5} \mathrm{Mpc}^{-3}$ ), and the surface brightness requirement further reduces their absolute number density by a factor of $\sim 6$. These objects tend to be much more luminous in the UV than typical local starburst galaxies studied previously (Heckman et al. 1998; Meurer et al. 1997, 1999) consistent with high SFRs and relatively little dust extinction. The median absolute UV magnitude of the sample is -20.3 , corresponding to $\simeq 0.5 L_{z=3}^{*}$, where $L_{z=3}^{*}$ is the characteristic luminosity of LBGs at $z \sim 3$
(Steidel et al. 1999; i.e., $M_{1700, A B}=-21.07$ ). Analysis of their spectra from the Sloan Digital Sky Survey (SDSS) and their spectral energy distributions (SEDs) from SDSS and GALEX subsequently showed that the LBAs are similar to LBGs in their basic global properties, including stellar mass, metallicity, dust extinction, SFR, physical size, and gas velocity dispersion. In Overzier et al. (2008), we showed that most of the UV emission in a preliminary sample of eight LBAs originate in highly compact burst regions in small, clumpy galaxies that are also morphologically similar to LBGs. We demonstrated that if LBGs at high redshift are also small merging galaxies similar to the LBAs; unfortunately, this would be very hard to detect given the much poorer physical resolution and sensitivity. Furthermore, for the present paper, it is important to point out that the LBAs also occupy an "offset" region in the main optical emission-line diagnostics diagram (i.e., $\log ([\mathrm{N} \mathrm{II}] / \mathrm{H} \alpha)$ versus $\log ([\mathrm{O} \mathrm{III}] / \mathrm{H} \beta)$, or the "BPT" (Baldwin et al. 1981) diagram) analogous to galaxies at high redshift. This suggests that similar physical conditions may apply to their interstellar medium (ISM) as well. In summary, the LBAs appear indeed good local analogs to the LBGs.

In this paper, we will present new results from our HST and Spitzer imaging campaigns and spectroscopic follow-up in order to highlight some of the remarkable properties of the LBA sample: we will compare the UV-optical sizes and morphologies, showing evidence for massive star-forming "clumps" that dominate the UV (Section 3). We explore possible connections between the starburst regions and the general properties of the optical emission-line gas (Section 4), as well as the relationship between the formation and evolution of massive stellar clumps in the context of the main structural components of young galaxies (Section 5). We conclude the paper with a summary and final remarks (Section 6). Forthcoming work on the LBA sample currently in preparation includes a detailed morphological analysis, multi-waveband modeling, mid-IR spectroscopy, very long baseline interferometry (VLBI) radio imaging, X-ray spectroscopy, and integral field and long-slit optical and near-IR spectroscopy.

\section{DATA}

\subsection{HST and Spitzer Imaging}

We observed 30 LBAs using HST (Programs 10920/11107, PI: Heckman). In order to probe morphologies in the UV, 24 objects were observed using the Advanced Camera for Surveys (ACS) Solar Blind Channel (SBC) in the filter F150LP $\left(\lambda_{c} \approx\right.$ $1614 \AA)$, six objects were observed using the high-resolution camera (HRC) in the filter F330W $\left(\lambda_{c} \approx 3334 \AA\right)$. The exposure times per object were typically $\sim 2500 \mathrm{~s}$. These UV images offer a tremendous gain in resolution over that of the GALEX images $\left(\approx 0\right.$. 07 compared to $\approx 5^{\prime \prime}$ for $\left.G A L E X\right)$. Matching observations in the rest-frame optical were carried out either using the Wide Field and Planetary Camera 2 (WFPC2) for $\sim 3600 \mathrm{~s}$ in F606W $\left(\lambda_{c} \approx 6001 \AA\right.$; twenty-four objects) or using the ACS Wide Field Channel for $\sim 2200 \mathrm{~s}$ in F850LP $\left(\lambda_{c} \approx 9170 \AA\right.$; seven objects). The ACS and WFPC2 observations were divided into, respectively, three and six dithered exposures and were combined using multidrizzle (Koekemoer et al. 2002).

We make use of photometric data obtained with the Multiband Imaging Photometer (MIPS) on the Spitzer Space Telescope at $24 \mu \mathrm{m}$ (Program ID 20390, PI: C. Hoopes). Photometry was obtained by performing point source extractions on the postbasic calibration data. 


\subsection{SDSS and VLT/FLAMES Spectroscopy}

We further use integrated optical emission-line fluxes and flux ratios measured from the SDSS spectra. In order to account for stellar absorption lines, the emission-line parameters were obtained from Gaussian fits after subtracting the continuum based on the best-fitting spectrum using stellar population models (see Tremonti et al. 2004).

The fraction of the total (line and continuum) light that is included within the $3^{\prime \prime}$ diameter SDSS fiber apertures is found to be $0.6 \pm 0.1$, estimated from the difference in fluxes measured in a $3^{\prime \prime}$ aperture compared to the total SDSS photometric aperture. ${ }^{10}$ We estimate that this estimate is in fact a lower limit to the fraction of UV and line emission included within the SDSS fiber aperture, based on the compactness of the starforming regions in the HST images and follow-up spectra.

In Section 4.1, we present a preview of data ${ }^{11}$ taken with the Fiber Large Array Multi-Element Spectrograph (FLAMES) on the European Southern Observatory (ESO) Very Large Telescope (VLT) during the night of 2008 October 30. We used the GIRAFFE/ARGUS integral field spectrograph (IFS) to obtain seeing-limited spectra resolving the $\mathrm{H} \alpha$ and $[\mathrm{N}$ II] $\lambda \lambda 6548 \AA$, $6584 \AA$ line complex at a spectral resolution of $R \simeq 9000$ and a spatial plate scale of 0.3 or 0.52 pixel $^{-1}$ (depending on the seeing). Each source was observed in four exposures of $400 \mathrm{~s}$ each. The data were pipeline reduced and further cleaned of cosmic rays and the background using our own routines.

More details on the LBA sample selection, observations and all measurements used in this paper are given elsewhere (Paper I; Hoopes et al. 2007; Basu-Zych et al. 2007, R. A. Overzier et al., 2009, in preparation). The object coordinates, redshifts, and basic quantities used in this paper are listed in Table 1. Emission-line measurements are listed in Table 2.

\subsection{Measuring Stellar Masses}

We will use stellar mass estimates that were obtained from the SDSS/DR7 stellar mass catalog. ${ }^{12}$ These masses are derived from fits to the SDSS photometry using a large grid of models constructed using the Bruzual \& Charlot (2003, BC03) stellar synthesis library. Although the BC03 models may not include the most up-to-date treatment of the thermally pulsating (TP)AGB phase, it has been shown that this phase mostly affects the stellar mass-to-light ratio in the rest-frame near-infrared $H$ and $K$ bands (e.g., see Eminian et al. 2008). Because near-infrared data are not being used in the fits, we believe that our stellar mass estimates are relatively accurate. However, in order to further reduce the uncertainty in these galaxy stellar mass estimates, we will derive a "best" mass by taking the average of the SDSS photometric mass and a dynamical mass that we calculate from the $\mathrm{H} \alpha$ emission-line velocity dispersion and the optical halflight radius. The two mass estimates typically agree to within $\sim 0.3$ dex with no systematic differences (see Table 1).

\subsection{Measuring Star Formation Rates}

We will make use of three different estimates of the integrated SFR. We use the most recent calibrations from literature as tabulated in Calzetti et al. (2009), and valid for a Kroupa (2008) initial mass function (IMF). The resulting SFRs are lower by a factor of $\sim 1.5$ compared to a Salpeter (1955) IMF.

\footnotetext{
${ }^{10}$ The fraction of fiber to total light in the SDSS $r^{\prime}$ band is estimated by $f_{\text {fiber }}=10^{-0.4\left(m_{r} \text {, fiber }-m_{r}, \text { Petro }\right)}$.

${ }_{11}$ ESO Program 082.B-0512(A).

12 Available at http://www.mpa-garching.mpg.de/SDSS/DR7/.
}

\subsection{1. $H \alpha$}

$\mathrm{SFR}_{\mathrm{H} \alpha, 0}$ is calculated from the $\mathrm{H} \alpha$ line luminosity and applying a correction for dust based on the Balmer decrement $\left(f_{\mathrm{H} \alpha} / f_{\mathrm{H} \beta}\right)$ following the recipe in (Calzetti 2001):

$$
\mathrm{SFR}_{\mathrm{H} \alpha, 0}\left[M_{\odot} \mathrm{yr}^{-1}\right]=5.3 \times 10^{-42} L_{\mathrm{H} \alpha, 0}\left[\mathrm{erg} \mathrm{s}^{-1}\right],
$$

where $L_{\mathrm{H} \alpha, 0}=L_{\mathrm{H} \alpha, \mathrm{obs}} 10^{0.4 A_{\mathrm{H} \alpha}}$, and

$$
\begin{aligned}
A_{\mathrm{H} \alpha} & =k(\mathrm{H} \alpha) E(B-V)_{\mathrm{gas}}, \\
E(B-V)_{\mathrm{gas}} & =\frac{\log _{10}\left[\left(f_{\mathrm{H} \alpha} / f_{\mathrm{H} \beta}\right) / 2.87\right]}{0.4[k(\mathrm{H} \alpha)-k(\mathrm{H} \beta)]},
\end{aligned}
$$

with $k(\mathrm{H} \alpha)=2.468$ and $k(\mathrm{H} \alpha)-k(\mathrm{H} \beta)=1.17$. We apply a small correction factor of typically $\sim 1.7$ due to the flux expected outside the SDSS fiber.

$$
\text { 2.4.2. } \mathrm{H \alpha}+24 \mu \mathrm{m}
$$

An improvement to the above method is given by $\mathrm{SFR}_{\mathrm{H} \alpha+24}$, which is based on a combination of the uncorrected $\mathrm{H} \alpha$ luminosity $\left(L_{\mathrm{H} \alpha \text {,obs }}\right)$, and the $24 \mu \mathrm{m}$ luminosity $\left(L_{24} \equiv v_{24} l_{24}\right)$ related to the emission of dust heated by young stars. It has been shown that $\mathrm{SFR}_{\mathrm{H} \alpha+24}$ is a robust indicator for the total SFR calibrated against $\mathrm{Pa} \alpha$ measurements in a large sample of nearby galaxies (Calzetti et al. 2007):

$$
\begin{aligned}
\operatorname{SFR}_{\mathrm{H} \alpha+24}\left[M_{\odot} \mathrm{yr}^{-1}\right]= & 5.3 \times 10^{-42} \\
& \times\left(L_{\mathrm{H} \alpha, \mathrm{obs}}+0.031 L_{24}\right)\left[\mathrm{erg} \mathrm{s}^{-1}\right] .
\end{aligned}
$$

As the exact, individual infrared SEDs are currently uncertain, we choose not to apply any $K$-corrections to the observed $24 \mu \mathrm{m}$ fluxes. Based on the average corrections found for a collection of spectral templates presented by Rieke et al. (2009, see their Figure 9), we estimate that the actual SFRs could be between 0.0 and 0.3 dex higher depending on the redshift, spectral shape, and total IR luminosity.

\subsubsection{FUV}

We will make use of $\mathrm{SFR}_{\mathrm{FUV}, 0}$, which is based on the dustcorrected far-UV luminosity using the empirical correlation between the attenuation of the UV and the IR emission from dust heated by obscured stars (the " $\beta-I R X$ relation," Meurer et al. 1995). The correlation has been shown to work well for relatively unobscured star-forming and starburst galaxies at both low and high redshifts (e.g., Meurer et al. 1995, 1999; Seibert et al. 2005; Reddy et al. 2006; Salim et al. 2007), but it must be noted that the situation is more complex for galaxies that have more complicated star formation histories (e.g., Kong et al. 2004; Johnson et al. 2007), very young ages (e.g., Reddy et al. 2006; Siana et al. 2008) or that are heavily obscured (e.g., Meurer et al. 1999; Goldader et al. 2002; Reddy et al. 2006). We use the calibration between UV color and $A_{\mathrm{FUV}}$ from Treyer et al. (2007), $A_{\mathrm{FUV}}=4.05\left(m_{\mathrm{FUV}}-m_{\mathrm{NUV}}\right)-0.18 \mathrm{mag}$, finding:

$$
\begin{aligned}
\mathrm{SFR}_{\mathrm{FUV}}\left[\mathrm{M}_{\odot} \mathrm{yr}^{-1}\right]= & 8.1 \times 10^{-29} \\
& \times l_{1500 \AA} 10^{0.4 A_{\mathrm{FUV}}}\left[\mathrm{erg} \mathrm{s}^{-1} \mathrm{~Hz}^{-1}\right] .
\end{aligned}
$$

Using the spectral slope $\beta=2.32 \log _{10}\left(m_{\mathrm{FUV}}-m_{\mathrm{NUV}}\right)-2.0$ with $f_{\lambda} \propto \lambda^{\beta}$ we further applied a small $K$-correction to obtain the monochromatic luminosity at rest-frame $1500 \AA, l_{1500 \AA}$. 
Table 1

Properties of the Lyman Break Analogs Sample

\begin{tabular}{|c|c|c|c|c|c|c|c|c|c|c|c|c|c|c|}
\hline \multirow[t]{2}{*}{ ID } & \multirow[t]{2}{*}{$\alpha_{J 2000}$} & \multirow[t]{2}{*}{$\delta_{J 2000}$} & \multirow[t]{2}{*}{$z$} & \multirow[t]{2}{*}{$\begin{array}{r}\log M_{*} \\
\left(M_{\odot}\right)\end{array}$} & \multirow[t]{2}{*}{$Z^{\mathrm{f}}$} & \multicolumn{3}{|c|}{$\begin{array}{l}\text { Star Formation Rate } \\
\qquad\left(M_{\odot} \mathrm{yr}^{-1}\right)\end{array}$} & \multirow[t]{2}{*}{$\begin{array}{c}\log L_{24} \\
\left(L_{\odot}\right)\end{array}$} & \multirow[t]{2}{*}{$\begin{array}{c}R_{e}^{\mathrm{a}} \\
(\mathrm{kpc})\end{array}$} & \multirow[t]{2}{*}{$C^{\mathrm{b}}$} & \multirow[t]{2}{*}{$\begin{array}{c}\sigma_{v}^{\mathrm{c}} \\
\left(\mathrm{km} \mathrm{s}^{-1}\right)\end{array}$} & \multirow[t]{2}{*}{$\begin{array}{c}\log M_{\text {dyn }}{ }^{\mathrm{d}} \\
\quad\left(M_{\odot}\right)\end{array}$} & \multirow[t]{2}{*}{$\begin{array}{l}t_{\text {dyn }}{ }^{\mathrm{e}} \\
(\mathrm{Myr})\end{array}$} \\
\hline & & & & & & $\mathrm{H} \alpha, 0$ & $F U V, 0$ & $\mathrm{H} \alpha+24$ & & & & & & \\
\hline 001009 & 00:10:09.97 & 00:46:03.66 & 0.243 & 10.5 & 8.44 & 4.6 & 25.3 & 4.8 & 9.4 & 3.06 & 3.73 & 91 & 10.3 & 33 \\
\hline 001054 & $00: 10: 54.85$ & $00: 14: 51.35$ & 0.243 & $\ldots$ & 8.61 & 14.4 & 7.9 & 26.9 & 10.5 & 4.24 & 3.91 & 157 & 10.9 & 27 \\
\hline 004054 & $00: 40: 54.33$ & $15: 34: 09.66$ & 0.283 & 9.2 & 8.03 & 7.4 & 0.4 & 13.9 & 10.0 & 0.95 & 2.22 & 65 & 9.5 & 14 \\
\hline 005439 & $00: 54: 39.80$ & $15: 54: 46.93$ & 0.236 & 10.6 & 8.66 & 4.6 & 3.6 & 15.4 & 10.3 & 1.73 & 4.74 & 120 & 10.3 & 14 \\
\hline 005527 & $00: 55: 27.46$ & $00: 21: 48.71$ & 0.167 & 9.7 & 8.28 & 22.7 & 13.2 & 55.4 & 10.8 & 0.77 & 3.53 & 124 & 10.0 & 6 \\
\hline 015028 & $01: 50: 28.41$ & 13:08:58.40 & 0.147 & 10.3 & 8.39 & 19.4 & 17.4 & 50.7 & 10.8 & 1.83 & 4.39 & 103 & 10.2 & 17 \\
\hline 020356 & 02:03:56.91 & $-08: 07: 58.51$ & 0.189 & 9.4 & 8.21 & 11.1 & 5.2 & 14.7 & 10.0 & 1.61 & 2.91 & 77 & 9.9 & 21 \\
\hline 021348 & $02: 13: 48.54$ & 12:59:51.46 & 0.219 & 10.5 & 8.74 & 6.7 & 61.6 & 35.1 & 10.7 & 1.53 & 8.34 & 86 & 10.0 & 18 \\
\hline 032845 & 03:28:45.99 & $01: 11: 50.85$ & 0.142 & 9.8 & 8.34 & 6.7 & 4.3 & 8.7 & 9.8 & 1.82 & 3.34 & 83 & 10.0 & 22 \\
\hline 035733 & $03: 57: 34.00$ & $-05: 37: 19.70$ & 0.204 & 10.0 & 8.43 & 9.6 & 20.4 & 12.7 & 10.0 & 1.09 & 2.92 & 86 & 9.8 & 12 \\
\hline 040208 & 04:02:08.87 & $-05: 06: 42.06$ & 0.139 & 9.5 & 8.30 & 2.0 & 1.4 & 2.5 & 9.0 & 1.42 & 3.92 & 66 & 9.7 & 21 \\
\hline 080232 & 08:02:32.35 & $39: 15: 52.68$ & 0.267 & 10.7 & 8.65 & 21.3 & 75.7 & 30.4 & 10.5 & 3.80 & 4.87 & 75 & 10.2 & 50 \\
\hline 080844 & 08:08:44.27 & $39: 48: 52.36$ & 0.091 & 9.8 & 8.74 & 3.7 & 26.3 & 16.1 & 10.3 & 0.88 & 6.69 & 131 & 10.1 & 7 \\
\hline 082001 & 08:20:01.72 & $50: 50: 39.16$ & 0.217 & 9.8 & 8.15 & 20.3 & 8.7 & 40.0 & 10.6 & 1.52 & 2.84 & 91 & 10.0 & 17 \\
\hline 082550 & $08: 25: 50.95$ & $41: 17: 10.30$ & 0.156 & 9.9 & 8.37 & 5.5 & 7.8 & 7.0 & 9.7 & 1.56 & 2.64 & 94 & 10.0 & 17 \\
\hline 083803 & 08:38:03.73 & $44: 59: 00.28$ & 0.143 & 9.5 & 8.18 & 4.5 & 4.0 & 6.2 & 9.5 & 0.92 & 2.49 & 75 & 9.6 & 12 \\
\hline 092159 & $09: 21: 59.39$ & $45: 09: 12.38$ & 0.235 & 10.8 & 8.67 & 25.8 & 38.5 & 55.1 & 10.8 & 1.80 & 5.98 & 135 & 10.4 & 13 \\
\hline 092336 & $09: 23: 36.46$ & $54: 48: 39.25$ & 0.222 & 9.8 & 8.41 & 9.3 & 5.2 & 9.9 & 9.8 & 0.48 & 3.38 & 101 & 9.6 & 5 \\
\hline 092600 & 09:26:00.41 & $44: 27: 36.13$ & 0.181 & 9.1 & 8.09 & 13.2 & 3.9 & 17.0 & 9.9 & 1.09 & 3.27 & 95 & 9.9 & 11 \\
\hline 093813 & $09: 38: 13.50$ & $54: 28: 25.09$ & 0.102 & 9.4 & 8.19 & 13.0 & 4.3 & 19.8 & 10.2 & 0.92 & 4.59 & 98 & 9.8 & 9 \\
\hline 101211 & $10: 12: 11.18$ & $63: 25: 03.70$ & 0.246 & 9.8 & 8.36 & 4.3 & 12.0 & 6.2 & 9.6 & 0.89 & 3.74 & 79 & 9.6 & 11 \\
\hline 102613 & $10: 26: 13.97$ & $48: 44: 58.94$ & 0.160 & 9.8 & 8.27 & 7.0 & 6.9 & 9.9 & 9.8 & 1.99 & 2.93 & 70 & 9.6 & 28 \\
\hline 113303 & 11:33:03.80 & $65: 13: 41.31$ & 0.241 & 9.1 & 8.02 & 5.3 & 3.9 & 7.7 & 9.6 & 0.77 & 3.30 & 64 & 9.4 & 12 \\
\hline 124819 & $12: 48: 19.75$ & $66: 21: 42.68$ & 0.260 & 9.9 & 8.34 & 17.8 & 24.3 & 18.4 & 10.1 & 1.90 & 3.10 & 71 & 10.0 & 24 \\
\hline 135355 & $13: 53: 55.90$ & $66: 48: 00.59$ & 0.198 & 9.9 & 8.40 & 17.1 & 50.4 & 19.4 & 10.1 & 3.57 & 4.85 & 86 & 10.3 & 41 \\
\hline 143417 & $14: 34: 17.16$ & $02: 07: 42.58$ & 0.180 & 10.7 & 8.65 & 25.4 & 24.6 & 20.0 & 10.1 & 4.60 & 4.51 & 99 & 10.5 & 46 \\
\hline 210358 & 21:03:58.75 & $07: 28: 02.45$ & 0.137 & 10.9 & 8.70 & 41.3 & 6.9 & 108.3 & 11.1 & 2.70 & 5.76 & 173 & 10.8 & 15 \\
\hline 214500 & $21: 45: 00.26$ & 01:11:57.58 & 0.204 & 9.9 & 8.49 & 13.6 & 37.8 & 16.4 & 10.1 & 1.16 & 3.56 & 78 & 9.8 & 15 \\
\hline 231812 & $23: 18: 13.00$ & $00: 41: 26.10$ & 0.252 & 10.0 & 8.31 & 30.8 & 42.7 & 63.1 & 10.8 & 2.54 & 3.29 & 82 & 10.1 & 30 \\
\hline 232539 & $23: 25: 39.23$ & $00: 45: 07.25$ & 0.277 & 9.2 & 8.18 & 9.9 & 9.5 & 12.8 & 9.9 & 0.81 & 2.93 & 70 & 9.5 & 11 \\
\hline 235347 & $23: 53: 47.69$ & 00:54:02.08 & 0.223 & 9.5 & 8.11 & 6.9 & 2.1 & 9.9 & 9.8 & 1.31 & 2.27 & 68 & 9.7 & 18 \\
\hline
\end{tabular}

Notes.

a Optical half-light radius.

${ }^{\mathrm{b}}$ Optical concentration index.

${ }^{c}$ Gas velocity derived from the $\mathrm{H} \alpha$ emission line width.

${ }^{\mathrm{d}}$ Dynamical mass $M_{\mathrm{dyn}}=3.4 R_{e} \sigma_{v}^{2} / G$.

e Dynamical time $t_{\mathrm{dyn}}=R_{e} / \sigma_{v}$

f Oxygen abundance in units of $12+\log (\mathrm{O} / \mathrm{H})$ estimated using the "O3N2" estimator from Pettini \& Pagel (2004).

\section{RESULTS}

\subsection{UV-Optical Morphologies}

In Figure 1, we show $6^{\prime \prime} \times 6^{\prime \prime} \mathrm{UV}$-optical false-color postage stamps of 30 LBAs, including the eight objects studied in Paper I. In all cases, both the UV and the optical lights are dominated by the intense light from young massive stars in very compact starburst regions. The optical images furthermore allow us to see the faint emission from older stars displaying a wide range of (often complex) morphologies, which include clumpy or chain-like galaxies, interacting pairs, a ring galaxy, and perturbed (spiral) disks. In Figure 2, we plot the half-light radii versus the stellar masses. The global median half-light radii of the galaxies are $\approx 1 \mathrm{kpc}$ and $\approx 1.5 \mathrm{kpc}$ in the UV and optical, respectively. The galaxy mass estimates range from a few times $10^{9}$ to $\lesssim 10^{11} M_{\odot}$ (Table 1$)$. The sizes and the stellar masses of these galaxies correspond well to those of typical LBGs (e.g., Shapley et al. 2001; Papovich et al. 2001; Giavalisco 2002; Ferguson et al. 2004; Stark et al. 2009).

As noted already in Paper I, a significant fraction of both the UV and optical emission are seen to come from luminous, very compact clumps. The much larger size of the sample presented here allows us to investigate this in more detail. In Figures 3 and 4, we show some close-ups of the rest-frame optical HST images at high contrast. LBAs typically consist of a collection of centrally located compact clumps having a range in magnitudes (e.g., Figure 3). However, in a minority of cases, the LBA contains a single, dominant luminous point-like source at or near the center of the galaxy (i.e., 005439, 021348, 080232, 080844, 092159, and 210358). In these sources, the central source typically contains $\sim 50 \%$ ( 20 and $77 \%$ being the lowest and the highest, respectively) of all the UV light. In the optical, the contribution from the host galaxies is larger, somewhat reducing the light fraction of the central source to $\sim 20 \%-30 \%$. Here we have used an aperture of $0{ }^{\prime} .2$ radius for the central point-like source and an aperture of $10^{\prime \prime}$ to estimate the total light of the galaxy. Because of their visual prominence and in anticipation of some of their remarkable properties that will be investigated in the following sections, we will henceforth refer to this subset of the clumps as "dominant central objects" (DCOs). A collage of images of the six objects hosting DCOs is shown in Figure 4. 
Table 2

Emission Line Ratios and Electron Density

\begin{tabular}{|c|c|c|c|c|c|c|c|}
\hline ID & $\log ([\mathrm{O} \mathrm{III}] / \mathrm{H} \beta)$ & $\log \left(\left[\mathrm{N}_{\mathrm{II}}\right] / \mathrm{H} \alpha\right)$ & $\log \left(\left[\mathrm{S} \mathrm{II}^{\mathrm{I}}\right] / \mathrm{H} \alpha\right)$ & $\log ([\mathrm{O} \mathrm{I}] / \mathrm{H} \alpha)$ & $\log \left(\left[\mathrm{N} \mathrm{II}_{\mathrm{II}}\right] /\left[\mathrm{S}_{\mathrm{II}}\right]\right)$ & {$\left[\mathrm{S}_{\text {II }}\right] \lambda 6713 \AA /\left[\mathrm{S}_{\text {II }}\right] \lambda 6731 \AA$} & $\begin{array}{c}N_{e} \\
\left(\mathrm{~cm}^{-3}\right) \\
\end{array}$ \\
\hline 001009 & 0.29 & -0.62 & $\ldots$ & -1.09 & $\ldots$ & $\ldots$ & $\ldots$ \\
\hline 001054 & 0.19 & -0.19 & -0.74 & -1.98 & 0.55 & 1.45 & 40 \\
\hline 004054 & 0.76 & -1.43 & -1.07 & -1.60 & -0.35 & 1.05 & 577 \\
\hline 005439 & -0.09 & -0.31 & -0.62 & -1.98 & 0.32 & 1.22 & 276 \\
\hline 005527 & 0.56 & -0.84 & -0.87 & -1.56 & 0.03 & 1.10 & 485 \\
\hline 015028 & 0.36 & -0.67 & -0.70 & -1.56 & 0.02 & 1.31 & 169 \\
\hline 020356 & 0.53 & -1.08 & -0.75 & -1.60 & -0.33 & 1.37 & 109 \\
\hline 021348 & -0.11 & -0.09 & -0.63 & -1.19 & 0.54 & 1.19 & 323 \\
\hline 032845 & 0.41 & -0.82 & -0.70 & -1.63 & -0.12 & 1.33 & 154 \\
\hline 035733 & 0.21 & -0.74 & -0.63 & -1.54 & -0.11 & 1.32 & 157 \\
\hline 040208 & 0.41 & -0.93 & -0.57 & -1.48 & -0.36 & $\ldots$ & $\ldots$ \\
\hline 080232 & -0.12 & -0.37 & -0.54 & -1.43 & 0.17 & 1.33 & 147 \\
\hline 080844 & -0.24 & -0.18 & -0.82 & -1.71 & 0.63 & 0.74 & 1991 \\
\hline 082001 & 0.63 & -1.20 & -0.79 & -1.55 & -0.41 & 1.27 & 219 \\
\hline 082550 & 0.29 & -0.83 & -0.55 & -1.58 & -0.27 & 1.45 & 36 \\
\hline 083803 & 0.57 & -1.16 & -0.79 & -1.63 & -0.37 & 1.31 & 173 \\
\hline 092159 & -0.13 & -0.30 & -0.55 & -1.21 & 0.25 & 1.18 & 346 \\
\hline 092336 & 0.32 & -0.68 & -0.66 & -1.41 & -0.02 & 1.25 & 246 \\
\hline 092600 & 0.73 & -1.27 & -1.03 & -1.91 & -0.24 & 1.31 & 169 \\
\hline 093813 & 0.62 & -1.06 & -0.87 & -1.68 & -0.18 & 1.33 & 154 \\
\hline 101211 & 0.45 & -0.69 & -0.70 & -1.20 & 0.01 & 1.17 & 352 \\
\hline 102613 & 0.40 & -1.03 & -0.58 & -1.42 & -0.44 & 1.39 & 88 \\
\hline 113303 & 0.74 & -1.46 & -1.01 & -1.98 & -0.46 & 1.35 & 130 \\
\hline 124819 & 0.38 & -0.85 & -0.65 & -1.59 & -0.20 & 1.24 & 253 \\
\hline 135355 & 0.24 & -0.79 & -0.56 & -1.48 & -0.23 & 1.39 & 93 \\
\hline 143417 & -0.16 & -0.40 & -0.60 & -1.59 & 0.19 & 1.29 & 199 \\
\hline 210358 & -0.03 & -0.12 & -0.74 & -1.39 & 0.63 & 1.11 & 449 \\
\hline 214500 & 0.10 & -0.64 & -0.60 & -1.48 & -0.04 & 1.25 & 248 \\
\hline 231812 & 0.44 & -0.87 & -0.67 & -1.42 & -0.21 & 1.29 & 193 \\
\hline 232539 & 0.62 & -1.10 & -0.80 & -1.57 & -0.29 & 1.19 & 327 \\
\hline 235347 & 0.69 & -1.25 & -0.81 & -1.55 & -0.45 & 1.46 & 31 \\
\hline
\end{tabular}

\subsection{Color-Magnitude Diagrams: Estimated Clump Masses}

We have measured the magnitudes and colors of each of the clumps and DCOs identified by eye inside circular 0".4 diameter apertures. The backgrounds were determined inside a local annulus and subtracted. We did not make a correction for the (small) amount of flux expected to fall outside the aperture due to the $H S T$ point-spread function (PSF). In Figure 5, we show the color-magnitude diagrams for all objects for which two filters were available, either FUV and $V$ (shown on the left) or $U$ and $z$ (shown on the right). Open and filled squares indicate the measured values, while the dotted lines indicate the dust-corrected values based on the Balmer decrement $\left(f_{\mathrm{H} \alpha} /\right.$ $f_{\mathrm{H} \beta}$ ) using a constant relation between reddening of the gas and the stellar continuum ${ }^{13}$ (Calzetti 2001). Unfortunately, we need to assume that the Balmer decrement as measured from the integrated SDSS spectrum applies to the much smaller clumps as well, although this is not necessarily correct. The results are tabulated in Table 3 . The clumps are very luminous with a median absolute magnitude in $V$ (approximately rest-frame $B$ ) of $\simeq-20 \mathrm{mag}$, and very blue (FUV $-V \simeq 0.0$ ). The DCOs are the most luminous having $M_{B} \sim-20$ to -22 mag. The small gap in the magnitude histograms in the top panels of Figure 5 may suggest that the DCOs are not just a bright tail of the general clumps distribution, but perhaps a distinct class of object.

A comparison with Starburst99 5.1 (Vázquez \& Leitherer 2005) stellar synthesis models indicates that the stellar masses

${ }^{13} E(B-V)_{*}=0.44 E(B-V)_{\mathrm{gas}}$. of the clumps shown in Figure 5 are typically a few times $10^{7}$ to a few times $10^{8} M_{\odot}$, while the DCOs have typical masses near or in excess of $10^{9} M_{\odot}$ (see Table 3). It is important to note that these mass estimates are relatively insensitive to dust extinction, since the dust extinction vector runs nearly parallel to the age vector in these plots. As shown in Figure 6, the masses are relatively insensitive to the star formation history (SFH) that we adopt. The figure also illustrates that it is much less straightforward to determine the typical stellar age of the clumps without accurate information about the extinction and SFH. For our adopted extinction corrections, the instantaneous burst models imply ages in the range from $\sim 5$ to $100 \mathrm{Myr}$ (red points in Figure 6). Alternatively, if we adopt a continuous SFH, the ages would be substantially higher, in the range $\sim 100-1000$ Myr (blue points), while the SFRs would have to be on the order of $\sim 10 M_{\odot} \mathrm{yr}^{-1}$ in order to explain the high luminosity of the brightest clumps (left blue track in Figure 5). The masses would be $\sim 2 \times$ higher on average compared to those derived for instantaneous bursts (Table 3). However, there are several arguments to make in favor of an instantaneous SFH (or a SFH having a very short $e$-folding time). We note that at least three of the sources show prominent Wolf-Rayet features indicating the presence of very massive stars at a special phase only 3-4 Myr after the onset of a massive burst (e.g., Brinchmann et al. 2008b). This would favor the instantaneous burst model. Also, we note that the very compact sizes of these objects (and thus the short dynamical times) are consistent with a short formation time $\left(t_{\text {form }} \gtrsim t_{\text {dyn }}\right)$. 


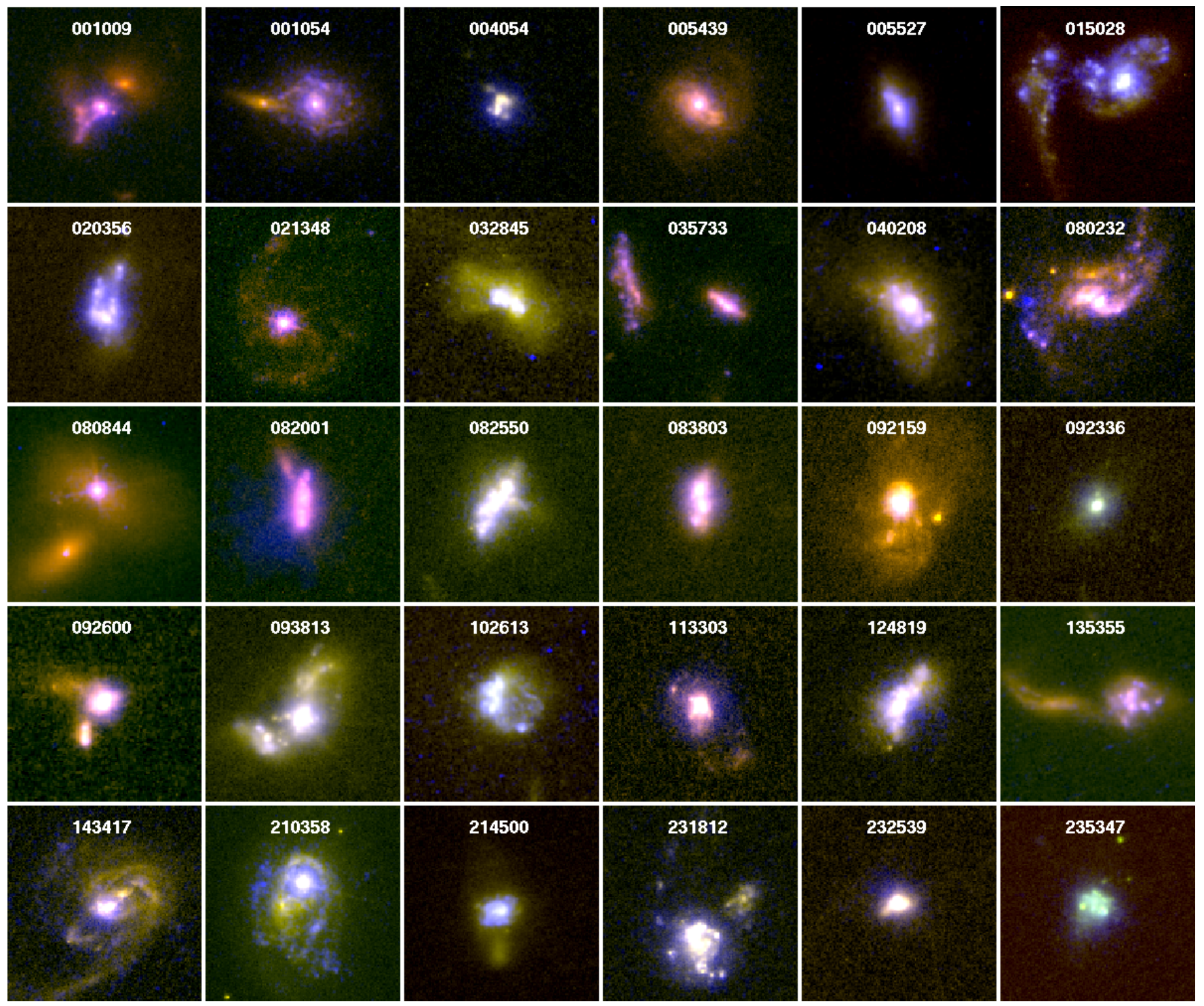

Figure 1. False-color HST images of the LBA sample showing the (rest-frame) UV in blue/purple and optical in yellow/red. The images measure $6^{\prime \prime} \times 6^{\prime \prime}$. The UV images were rebinned $(2 \times 2)$ to match the pixel scale of the optical images, and convolved with a Gaussian kernal with 0". 1 (FWHM). Although most objects are highly compact in both the UV and optical, a small subset consists of a very bright unresolved component in the middle of an extended, low surface brightness disk. The images demonstrate a wide range of complex morphologies often suggestive of interactions and (post-)merging. See the text for details. (A color version of this figure is available in the online journal.)
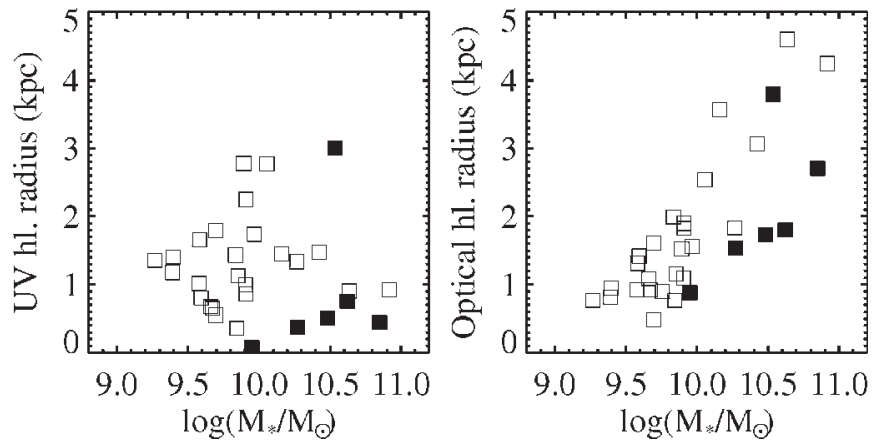

Figure 2. Stellar mass vs. the half-light radius measured in the UV (left panel) and optical (right panel) images. Solid squares mark the subset of DCOs in anticipation of results obtained in Section 5.2.

\subsection{Clump Sizes}

It is clear from simple visual inspection of the $H S T$ images that the DCOs are marginally resolved at best (note the strong diffraction spikes associated with the DCOs in Figure 3). Many of the other clumps appear either as faint point-like sources or larger spatially resolved regions (see examples in Figure 3).

To obtain the best constraints on the sizes of the DCOs, we have first examined the radial flux profile of the three brightest examples, using the ACS UV images as they have smaller pixels and a sharper PSF compared to the optical images. The result is shown in Figure 7. Each panel shows the measured count rate (plus signs) compared to the measured profile of a model PSF (dashed line) modeled using the TinyTim 3.0 software (Krist 1995). We also simulated a range of two-dimensional Gaussian models having a FWHM of 0''005-0'.125 and convolved them with the model PSF. The results are shown using colored lines. In all three cases, the data are most consistent with an object no larger than $\approx 0^{\prime} .075$ (FWHM), corresponding to effective radii no larger than $\simeq 70-160 \mathrm{pc}$ at $z=0.1-0.3$ ).

In order to be consistent across our entire clump sample and with our earlier definition of the clumps, we have measured the physical sizes of the brightest clumps in each LBA by measuring 

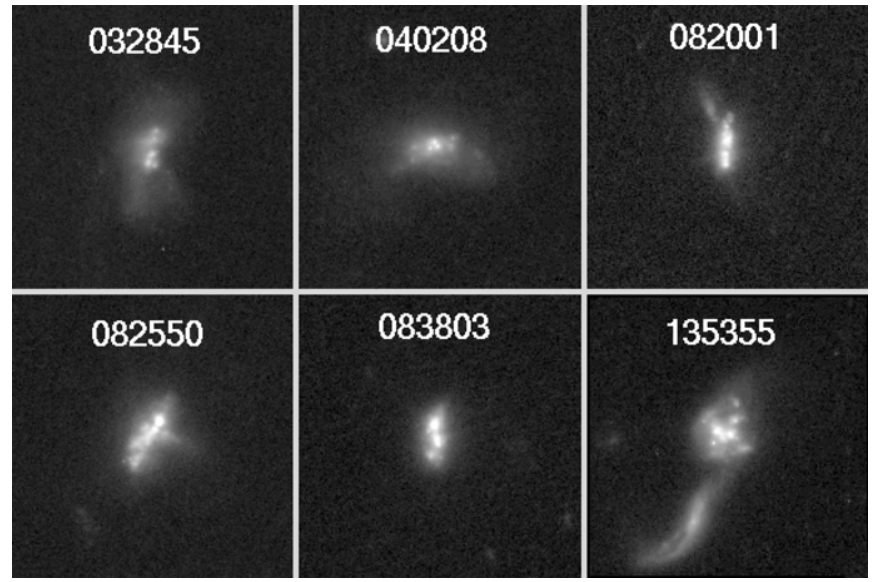

Figure 3. Illustration of HST optical morphologies of typical LBAs having several star-forming clumps of comparable brightness. These are to be contrasted with the six LBAs having a dominant compact central object shown in Figure 4. The images measure $10^{\prime \prime} \times 10^{\prime \prime}$.

the optical half-light radii of each clump within the same fixed angular apertures used in Figure 5. The radii were deconvolved assuming a PSF of 0 .'1 (FWHM). In Figure 8, we plot the clump radii versus the extinction-corrected clump absolute magnitudes. The typical clumps have radii of $\sim 200-400 \mathrm{pc}$, while the DCOs have radii of $\sim 100-200 \mathrm{pc}$. Comparing the sizes obtained here with those obtained for the three DCOs using our more careful model simulations shown in Figure 7 suggests that the more crude analysis may somewhat overestimate the true sizes of at least the DCOs. In any case, Figure 8, shows an inverse correlation between clump luminosity and size, largely defined by the extreme properties of the DCOs. Their high luminosities (corresponding to very high stellar masses) and compact sizes imply very high densities. We will discuss the implications of this remarkable result later in the paper.

\subsection{The Relation between Clumps and Their Host Galaxies}

We have demonstrated above that massive star-forming clumps are an essential ingredient in our sample of local LBG analogs, and have found that a subset contain a single extremely massive (up to several $\times 10^{9} M_{\odot}$ ) compact (radius $\sim 10^{2} \mathrm{pc}$ ) DCO. How do the properties of these compact structures relate to the overall properties of the host galaxies?

The results are shown in the six panels of Figure 9. In all panels, we plot the extinction-corrected absolute $B$-band magnitudes of the clumps measured within a fixed radius of $500 \mathrm{pc}$, in order to account for the effect that the angular sizes change by a factor of $\sim 2.5$ over the redshift range $z=0.1-0.3$. The results are as follows.

Galaxy concentration. In panel (a), we plot clump $M_{B}$ versus the optical concentration parameter ${ }^{14}$ of the host galaxy,

\footnotetext{
14 To calculate the concentration, we follow the procedures described in full detail in Lotz et al. (2006) and Paper I. First, we use SExtractor to make an object segmentation map and mask out neighboring objects. The image is background subtracted, and we calculate an initial Petrosian radius $\left(r_{P}\right.$ with $\eta \equiv 0.2$ ) using the object center and (elliptical) shape information from SExtractor. We then smooth the image by $\sigma=r_{P} / 5$ and create a new segmentation map by selecting those pixels that have a surface brightness higher than the mean surface brightness at the Petrosian radius. We recalculate the object center by minimizing the second-order moment of the flux, and then recalculate the Petrosian radius in the original image using this center. The total flux is defined as the flux within a radius of $1.5 \times r_{P}$, and $C$ is then calculated in circular apertures containing $20 \%$ and $80 \%$ of the light.
}

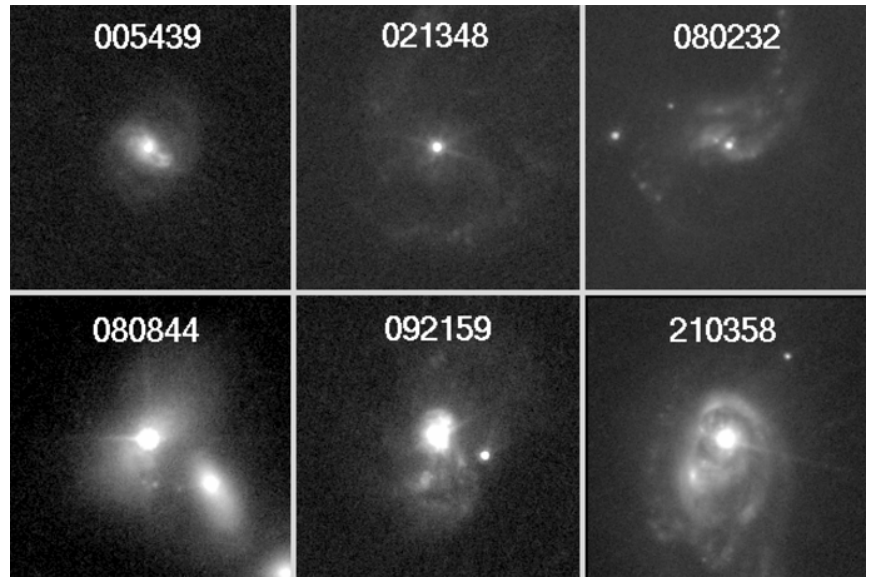

Figure 4. HST optical morphologies of the six LBAs having a dominant compact central object. The images measure $10^{\prime \prime} \times 10^{\prime \prime}$.

$C \equiv 5 \log \left(\frac{R_{80}}{R_{20}}\right)$. In general, the more luminous clumps occur in the LBAs with larger concentrations. The six DCOs (filled squares) are the most extreme. Inspection of Figure 1 shows that these objects are indeed systematically found inside larger, optically faint disks compared to the sample on average.

Galaxy half-light radius. In panel (b), we plot $M_{B}$ versus the half-light radii for each galaxy measured in the UV images. This plot shows that the total UV light in galaxies hosting a DCO is dominated by the DCO itself, albeit with one notable exception (DCO object 080232 having $R_{\mathrm{UV}} \sim 3 \mathrm{kpc}$ ).

Stellar mass. In panel (c), we plot $M_{B}$ versus the stellar mass of the hosts. The brighter clumps are predominantly found in the more massive objects in our sample. The DCOs are found in galaxies with masses $M_{*} \simeq 10^{10-11} M_{\odot}$.

Mid-IR luminosity. In panel (d), we show the relation between optical clump luminosity and the host galaxy luminosity at $24 \mu \mathrm{m}$. We find a correlation with $L_{24 \mu \mathrm{m}}$ indicating that the galaxies hosting the brighter clumps seen in the UV-optical are also the stronger IR sources. Even though selected on the basis of their high luminosity in the far-UV, the galaxies with DCOs have mid-IR luminosities similar to those of luminous infrared galaxies (LIRGs).

SFR. These high mid-IR luminosities imply high SFRs $\left(\mathrm{SFR}_{\mathrm{H} \alpha+24}\right)$, as shown in panel (e). The SFRs are systematically larger in the LBAs with the more luminous clumps, and the six galaxies with DCOs have SFRs ranging from 15 to nearly $100 M_{\odot} \mathrm{yr}^{-1}$.

Comparing SFR indicators. In panel (f), we plot $M_{B}$ versus the ratios of the two commonly used SFR indicators: $\mathrm{SFR}_{\mathrm{H} \alpha}$ and $\mathrm{SFR}_{\mathrm{H} \alpha, \mathrm{obs}+24}$. For the LBAs with the lower luminosity clumps, the majority show good agreement between the two SFR indicators. The LBAs with the brighter clumps are systematically displaced toward low values of $\mathrm{SFR}_{\mathrm{H} \alpha, 0} / \mathrm{SFR}_{\mathrm{H} \alpha, \mathrm{obs}+24}$, with the galaxies having a DCO being among the most extreme. To further investigate the discrepancy between $\mathrm{SFR}_{\mathrm{H} \alpha, 0}$ and $\mathrm{SFR}_{\mathrm{H} \alpha, o b+24}$ for the brightest sources, we compare $\mathrm{SFR}_{\mathrm{H} \alpha, 0} /$ $\mathrm{SFR}_{\mathrm{H} \alpha, \mathrm{obs}+24}$ with the ratio $\mathrm{SFR}_{\mathrm{H} \alpha, 0} / \mathrm{SFR}_{\mathrm{FUV}, 0}$. The result is shown in Figure 10 comparing the three SFR indicators. For the majority of sources, all the three indicators agree to within a factor of $\sim 2$ to 3 , but there are outliers for which the SFRs derived from the extinction-corrected $\mathrm{H} \alpha$ luminosity are significantly 

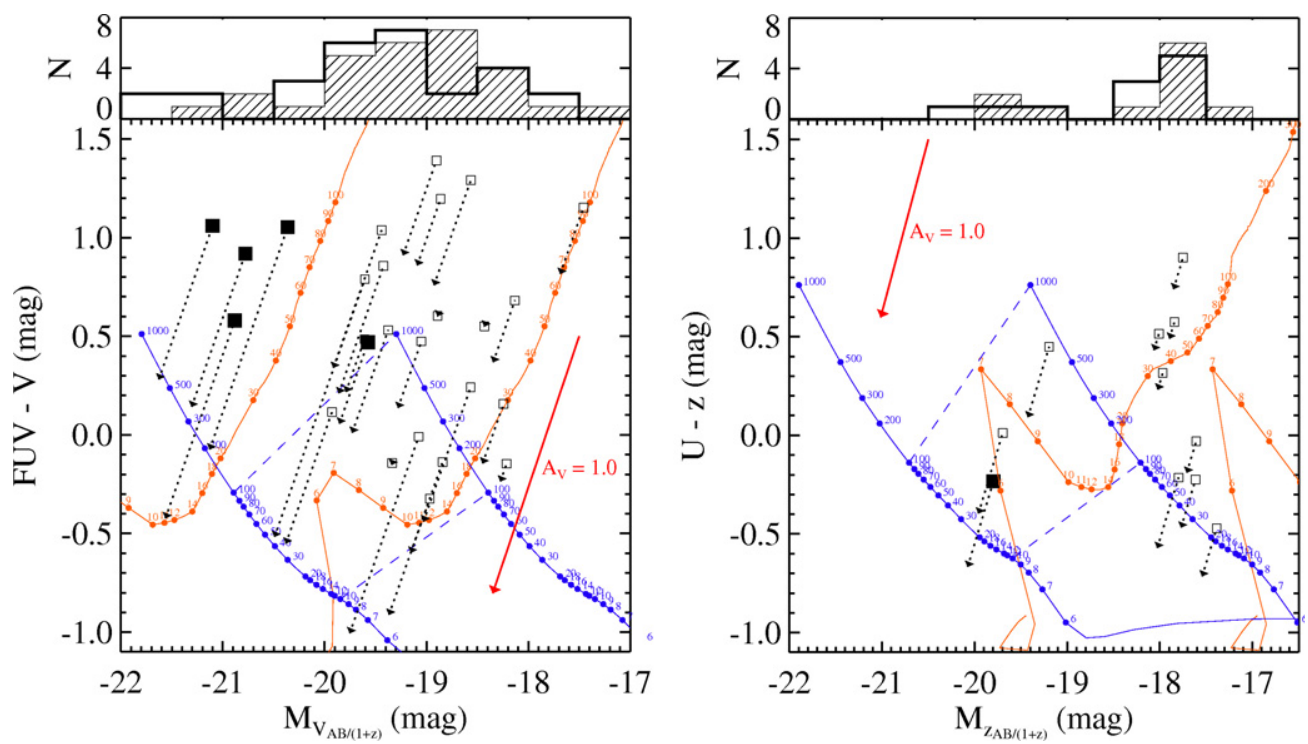

Figure 5. Top panel: Histogram of the absolute magnitude distribution corresponding to the clumps shown in the bottom panel. The measured (dust-corrected) distribution is indicated by the shaded (open) histogram. Typical massive star clusters in nearby galaxies have $M_{V} \simeq-(16-14)$ that would be as bright as $M_{V} \simeq-(19-17)$ when observed at the young age of $\sim 10$ Myr. The LBA clumps, and the DCOs in particular, are generally much brighter than typical massive star clusters. See Section 5 for discussion. Bottom panel: color-magnitude diagram of star-forming clumps identified in the HST WFPC2 images. Solid points show measured values, while dotted lines connecting to open points indicate dust-corrected values based on the Balmer decrement and assuming a constant relation between reddening of the gas and the stellar continuum following Calzetti (2001). Colored lines indicate population synthesis tracks modeled using Starburst99 for either constant bursts with a SFR of 1 and $10 M_{\odot} \mathrm{yr}^{-1}$ (right and left blue tracks) or an instantaneous burst normalized to a total stellar mass of $10^{8} M_{\odot}$ and $10^{9} M_{\odot}$ (right and left red tracks). Ages in Myr have been indicated along the tracks. Blue dashed lines indicate the ages at which the constant burst models reach a total stellar mass of $10^{8} M_{\odot}$ and $10^{9} M_{\odot}$. The models used have a LMC metallicity, include the nebular continuum, and were redshifted to $z=0.15$. The red arrow indicates the reddening vector corresponding to an extinction of $A_{V}=1.0 \mathrm{mag}$. The six DCOs are indicated by the filled squares.

(A color version of this figure is available in the online journal.)

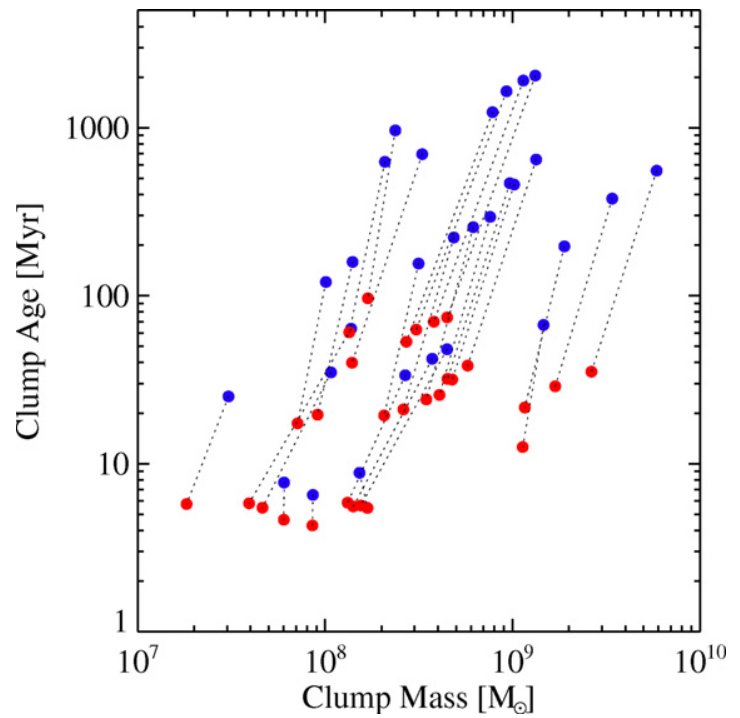

Figure 6. Clump masses and ages estimated from the color-magnitude diagrams presented in Figure 5, assuming either an instantaneous star formation history (red points) or a continuous SFH (blue points). The masses estimated using the continuous model are only $\sim 2 \times$ higher on average compared to those derived for instantaneous bursts, while the ages estimated using the continuous model are significantly higher compared to the instantaneous model.

(A color version of this figure is available in the online journal.)

lower than either both or one of the other two SFRs. In other words, the ratio of the dust-corrected $\mathrm{H} \alpha$ luminosity relative to the luminosities of the mid-IR and (intrinsic) far-UV continua is systematically small in the galaxies with DCOs compared to normal star-forming galaxies and the less extreme LBAs. We will refer to these offsets in Sections 4.1 and 4.2 below.

\section{DISCUSSION OF THE EMISSION-LINE PROPERTIES OF LBAs}

The most significant results provided by our $H S T$ data are as follows.

1. Highly luminous, compact star-forming "clumps" appear to be the defining feature of the UV morphology of the LBAs.

2. In the most extreme cases, the UV images are dominated by a single extremely bright and compact object surrounded by a disturbed envelope or disk. We have called these DCOs.

3. The SFR derived from the extinction-corrected $\mathrm{H} \alpha$ emission-line luminosity tends to be systematically smaller than that derived using the mid-IR and/or the extinctioncorrected far-UV continuum luminosities (see panel (f) of Figures 9 and 10). More properly, the ratio of the $\mathrm{H} \alpha$ luminosity to either the mid-IR or far-UV continuum is smaller on average than in typical star-forming galaxies.

For our discussion below, it is important to address first whether any of these properties could be explained by the contribution from a Type 1 (unobscured) or Type 2 (obscured) AGN.

\subsection{Are They AGNs?}

\subsubsection{Type 1 AGNs}

Could the DCOs be Type 1 AGNs in which we have a direct view of the accretion disk and broad emission-line region? This would account for the exceptionally compact morphology as well as for the significant $24 \mu \mathrm{m}$ "excess." We note that the initial LBA selection process excluded any obvious Type I AGN in the first place based on the absence of obvious broad (several thousand $\mathrm{km} \mathrm{s}^{-1}$ ) Balmer emission lines from the AGN 
Table 3

Properties of the Clumps

\begin{tabular}{|c|c|c|c|c|c|c|c|c|}
\hline $\mathrm{ID}^{\mathrm{a}}$ & $\#$ & $\begin{array}{c}\text { Abs. Mag }{ }^{\mathrm{c}} \\
(\mathrm{mag})\end{array}$ & $\begin{array}{c}\text { Color }^{\mathrm{d}} \\
(\mathrm{mag})\end{array}$ & $\begin{array}{c}\operatorname{Age}_{s b}{ }^{\mathrm{e}} \\
(\mathrm{Myr})\end{array}$ & $\begin{array}{c}\log M_{*, \mathrm{sb}}{ }^{\mathrm{e}} \\
\left(M_{\odot}\right) \\
\end{array}$ & $\begin{array}{r}\operatorname{Age}_{\mathrm{cst}}{ }^{\mathrm{f}} \\
(\mathrm{Myr})\end{array}$ & $\begin{array}{c}\log M_{*, \mathrm{cst}} \mathrm{f} \\
\left(M_{\odot}\right)\end{array}$ & $\begin{array}{c}\mathrm{SFR}_{\mathrm{cst}}{ }^{\mathrm{f}} \\
\left(M_{\odot} \mathrm{yr}^{-1}\right)\end{array}$ \\
\hline 210358 & 1 & -21.31 & 0.14 & 29 & 9.23 & 378 & 9.53 & 9.1 \\
\hline 021348 & 1 & -21.63 & 0.28 & 35 & 9.42 & 555 & 9.77 & 10.6 \\
\hline 092159 & 1 & -21.56 & -0.42 & 13 & 9.05 & 67 & 9.17 & 21.9 \\
\hline 080232 & 1 & -21.13 & -0.07 & 22 & 9.07 & 197 & 9.28 & 9.7 \\
\hline $080844^{\mathrm{b}}$ & 1 & -20.08 & -0.67 & 5 & 8.23 & 9 & 8.18 & 17.3 \\
\hline 214500 & 1 & -19.91 & 0.34 & 38 & 8.76 & 647 & 9.13 & 2.1 \\
\hline 082550 & 1 & -18.93 & 0.76 & 63 & 8.49 & 1646 & 8.97 & 0.6 \\
\hline 082001 & 1 & -19.23 & 0.91 & 74 & 8.65 & 2044 & 9.12 & 0.7 \\
\hline 124819 & 1 & -19.86 & 0.21 & 32 & 8.68 & 459 & 9.01 & 2.3 \\
\hline 005439 & 1 & -19.85 & 0.06 & 26 & 8.61 & 295 & 8.88 & 2.6 \\
\hline 232539 & 1 & -19.79 & 0.22 & 32 & 8.65 & 467 & 8.99 & 2.1 \\
\hline 231812 & 1 & -19.09 & 0.86 & 70 & 8.58 & 1909 & 9.06 & 0.6 \\
\hline $135355^{b}$ & 1 & -19.42 & 0.09 & 21 & 8.42 & 222 & 8.69 & 2.2 \\
\hline $040208^{b}$ & 1 & -18.06 & 0.43 & 53 & 8.16 & 560 & 8.36 & 0.5 \\
\hline 001009 & 1 & -19.73 & 0.01 & 24 & 8.54 & 256 & 8.79 & 2.5 \\
\hline $040208^{b}$ & 2 & -17.90 & 0.49 & 60 & 8.13 & 626 & 8.32 & 0.4 \\
\hline $102613^{b}$ & 1 & -17.85 & 0.74 & 96 & 8.23 & 964 & 8.37 & 0.3 \\
\hline 231812 & 2 & -19.28 & 0.14 & 29 & 8.41 & 372 & 8.72 & 1.4 \\
\hline $005527^{\mathrm{b}}$ & 1 & -19.96 & -0.40 & 6 & 8.12 & 34 & 8.43 & 8.0 \\
\hline 004054 & 2 & -18.89 & 0.60 & 53 & 8.43 & 1237 & 8.89 & 0.7 \\
\hline 113303 & 1 & -19.34 & -0.15 & 19 & 8.31 & 155 & 8.50 & 2.1 \\
\hline $040208^{b}$ & 3 & -18.02 & 0.23 & 26 & 7.92 & 346 & 8.24 & 0.6 \\
\hline 093813 & 1 & -19.15 & -0.60 & 5 & 7.67 & 35 & 8.03 & 3.1 \\
\hline 083803 & 1 & -18.27 & -0.24 & 17 & 7.85 & 121 & 8.00 & 0.9 \\
\hline 092336 & 1 & -20.38 & -0.55 & 6 & 8.15 & 42 & 8.57 & 8.9 \\
\hline 235347 & 1 & -18.34 & 0.37 & 40 & 8.14 & 695 & 8.52 & 0.5 \\
\hline 001054 & 1 & -20.49 & -0.52 & 6 & 8.19 & 48 & 8.65 & 9.4 \\
\hline 004054 & 1 & -18.43 & 0.55 & 50 & 8.24 & 1101 & 8.68 & 0.5 \\
\hline 143417 & 1 & -19.75 & -1.00 & 4 & 7.93 & 7 & 7.93 & 13.2 \\
\hline 020356 & 1 & -18.45 & -0.14 & 20 & 7.96 & 159 & 8.15 & 0.9 \\
\hline 015028 & 1 & -19.37 & -0.91 & 5 & 7.78 & 8 & 7.78 & 7.9 \\
\hline 231812 & 3 & -17.69 & 0.82 & 67 & 8.01 & 1796 & 8.49 & 0.2 \\
\hline 035733 & 1 & -19.03 & -0.44 & 6 & 7.59 & 64 & 8.14 & 2.2 \\
\hline $032845^{b}$ & 1 & -17.77 & -0.47 & 6 & 7.26 & 25 & 7.48 & 1.3 \\
\hline $032845^{\mathrm{b}}$ & 2 & -17.54 & -0.71 & 5 & 7.22 & 8 & 7.17 & 1.9 \\
\hline $135355^{b}$ & 2 & -18.03 & -0.57 & 6 & 7.39 & 15 & 7.47 & 2.0 \\
\hline $102613^{b}$ & 2 & -17.71 & -0.19 & 6 & 7.18 & 83 & 7.76 & 0.7 \\
\hline
\end{tabular}

Notes.

${ }^{\mathrm{a}}$ ID number of the host galaxy of each clump.

${ }^{\mathrm{b}}$ Magnitudes and colors correspond to those measured in the filters F850LP and F330W-F850LP, instead of, F606W and F150LP-F606W, respectively.

${ }^{\mathrm{c}}$ Absolute (optical) clump magnitude from Figure 5, $M=m_{\mathrm{cor}}-5 \log \left(\frac{D_{L}}{10 \mathrm{pc}}\right)+2.5 \log (1+z)$, where $D_{L}$ is the luminosity distance. $m_{\text {cor }}$ is the apparent magnitude corrected for dust using $m_{\text {cor }}=m-E(B-V)_{*} k^{\prime}(\lambda)$ and assuming $E(B-V)_{*}=0.44 E(B-V)_{\text {gas }}$ (Calzetti 2001).

${ }^{\mathrm{d}}$ Dust-corrected clump color from Figure 5.

${ }^{\mathrm{e}}$ Clump age and stellar mass under the assumption of the instantaneous star formation history shown in Figure 5 (red tracks).

${ }^{\mathrm{f}}$ Clump age, stellar mass, and SFR under the assumption of the continuous star formation history shown in Figure 5 (blue tracks).

broad-line region in the SDSS spectra (Hoopes et al. 2007). We can quantify this and exclude the possibility that some of our galaxies are of type Seyfert 1.8 or 1.9, with weak broad emission lines. In the event that possible broad emission was somehow being missed in the integrated SDSS spectrum, we have obtained integral field spectra using FLAMES on the VLT. In Figure 11, we show the preliminary results of the $\mathrm{H} \alpha+[\mathrm{N}$ II $]$-line profile as measured in the central $\sim 1^{\prime \prime}$ region centered on the clumps in four LBAs hosting some of the most luminous clumps (three of which are DCOs). The line profiles were fitted with a series of narrow and broad Gaussian components indicated by red and blue dashed lines, respectively. The fits show that each source has significant line asymmetries on the blue side of the narrow lines that are consistent with a relatively broad, blueshifted component (R. A. Overzier et al., 2009, in preparation). Such blue asymmetries of width of a few hundred $\mathrm{km} \mathrm{s}^{-1}$ in both the permitted and forbidden are not related to the much broader line profiles from Type 1 AGNs, which are seen only for the permitted lines. Instead, the broad blueshifted wings seen on the emission-line profiles in our objects (Figure 11) are signposts of galactic winds and are commonly observed in intense local and high-redshift starbursts (e.g., Heckman et al. 1990; Lehnert \& Heckman 1996; Lehnert et al. 2009; Shapiro et al. 2009).

\subsubsection{Type 2 AGNs}

Our spectra show clearly that the optical continuum must be dominated by the light of young stars (with no detectable contribution from an unobscured AGN). However, is it possible that the DCOs are associated with the presence of an obscured (Type 2) AGN? In Figure 12, we show the $\log ([\mathrm{N}$ II $] / \mathrm{H} \alpha)$ versus 

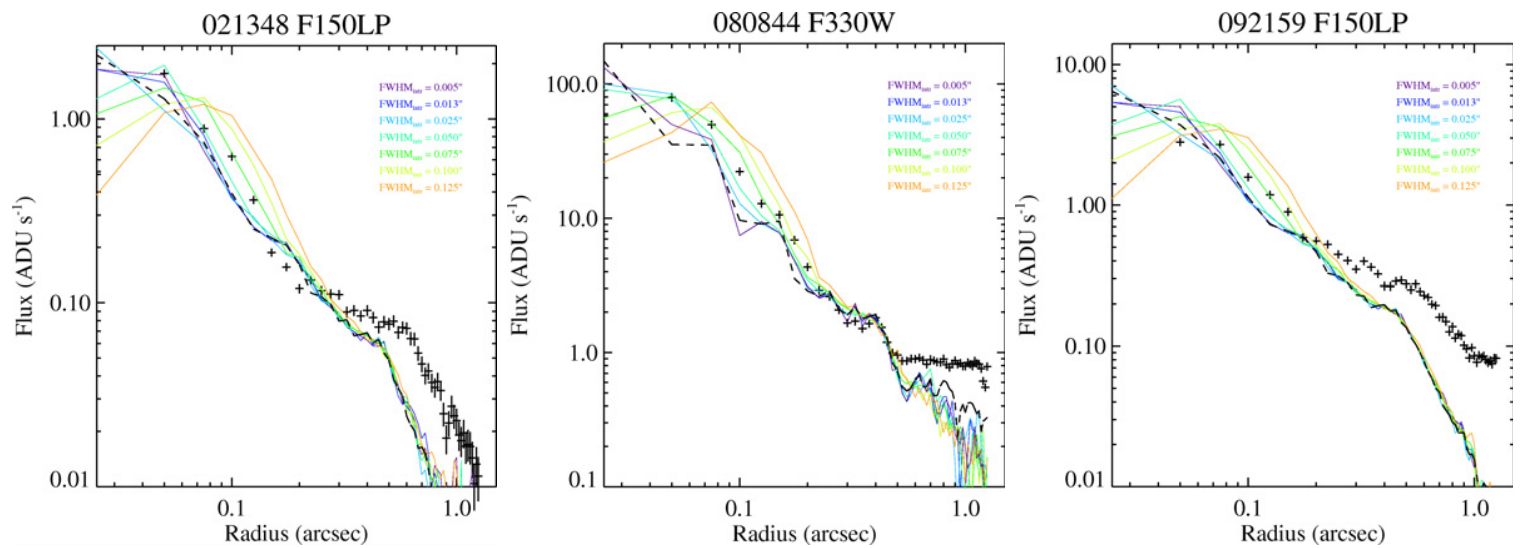

Figure 7. Radial flux profiles of the three strongest, point-like sources identified in the HST images. Panels show the measured count rates (plusses) in either the ACS/SBC or HRC images compared to the measured profile of a model PSF (dashed line) modeled using the TinyTim 3.0 software (Krist 1995). To investigate the size limits we can place on the nuclear sources, we simulated a range of 2D Gaussian models having a FWHM of 0'.005-0'.125 and convolved them with the model PSF. The results are shown using colored lines. In all three cases, the data is most consistent with an object no larger than $\approx 0$ '”075 (FWHM; corresponding to $135-$ $320 \mathrm{pc}$ at $z=0.1-0.3$ ).

(A color version of this figure is available in the online journal.)

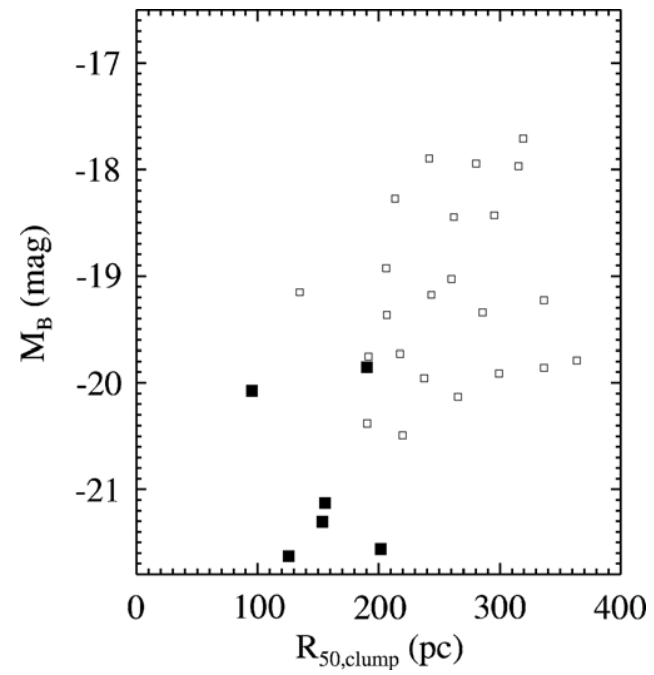

Figure 8. Absolute magnitude vs. the optical half-light radius of the brightest clump in each galaxy estimated in Section 3.3. The six DCOs are indicated by filled squares.

the $\log ([\mathrm{O} \mathrm{III}] / \mathrm{H} \beta)$ diagnostic "BPT" diagram that is most commonly used for identifying the main source of ionization among samples of star-forming galaxies and AGN (Baldwin et al. 1981; Kauffmann et al. 2003; Kewley et al. 2006). Because it is based on ratios of bright emission lines that are observable out to $z \lesssim 3$, the diagram is widely used to characterize galaxies at both low and high redshift. It has been known for some time that intensely star-forming galaxies at high redshift such as Lyman breaks, "s $B z K$," and distant red galaxies are often peculiarly offset toward higher line ratios (e.g., Teplitz et al. 2000; Erb et al. 2006a; Kriek et al. 2007; Lehnert et al. 2009; Hayashi et al. 2009) with respect to the mean SDSS star-forming population at low redshift (Figure 12, dotted line). Similar offsets, albeit perhaps slightly less extreme, have been observed in local "warm" IR-luminous galaxies (Kewley et al. 2001) and Wolf-Rayet galaxies (Brinchmann et al. 2008b), and DEEP2 galaxies at $z \sim 1.4$ (Shapley et al. 2005; Liu et al. 2008). At present it is not known what physical mechanisms lie at the root of these offsets (see the detailed discussion in Brinchmann et al. 2008a), but it is important to note that our sample (filled circles) shows similar offsets in the BPT diagram (see also Hoopes et al. (2007) and Paper I). The BPT displacement is in the sense of enhancements in one or both of the two line ratios. If we focus on the locations of the host galaxies of the DCOs in Figure 12 (filled squares), we see that they are more significantly displaced (in $\log \left[\left[\mathrm{N}_{\mathrm{II}}\right] / \mathrm{H} \alpha\right]$ ) with respect to the locus of normal star-forming galaxies and the other LBAs. Typical galaxies in the SDSS with similar emission-line ratios are almost certainly composite objects in which the SDSS fiber encompasses both gas near the nucleus that is excited by an AGN and normal regions of star formation in the surrounding galaxy (e.g., Kauffmann et al. 2003; Kewley et al. 2006; Kauffmann \& Heckman 2009). Could the DCOs harbor a Type 2 AGN surrounded by a starburst?

To answer this question, we further investigate the BPT offsets in Figure 13. In panel (a), we plot for each clump the dimensionless parameter $d_{P}$, defined as the relative offset its host galaxy has in the BPT diagram. This offset distance $d_{P}$ is measured perpendicular to the SDSS star-forming "ridge" (dotted line in Figure 12). This clearly demonstrates a relation between the offsets and the luminosity of the clumps. Panel (b) shows the offsets along $\log ([\mathrm{NII}] / \mathrm{H} \alpha)$ only, but we note that the correlation here is driven mainly by the well-known relation between mass and metallicity. In panel (c), we plot $M_{B}$ versus the electron density $\left(N_{e}\right)$ of the gas as derived from the density sensitive [S II] $] \lambda 6713 \AA, 6731 \AA$ line doublet assuming a gas temperature of $10^{4} \mathrm{~K}$ (Osterbrock \& Ferland 2006). The densities derived from the integrated SDSS spectrum correlate with the $M_{B}$ of the clumps over almost two orders of magnitude (the lowest and the highest being $\sim 30$ and $\sim 2000 \mathrm{~cm}^{-3}$ ) albeit with quite a large scatter. The implied gas pressures are $P / k \sim 10^{6}$ to $10^{7} \mathrm{~K} \mathrm{~cm}^{-3}$, several orders of magnitude higher than in normal star-forming galaxies in the local universe.

The Type 2 AGN interpretation would be consistent with the high electron densities seen in the most extreme cases $\left(\sim 10^{3} \mathrm{~cm}^{-3}\right)$, since such densities are typical in the narrow emission-line region in the AGN (Osterbrock \& Ferland 2006). It would also be consistent with the blue-asymmetric emissionline profiles seen in Figure 11, which are characteristic of outflows in an emission-line AGN (e.g., Heckman et al. 1981; Whittle et al. 1988). The AGN hypothesis could also explain why the ratio of the 24 micron and extinction-corrected $\mathrm{H} \alpha$ luminosity is higher than in typical star-forming galaxies 

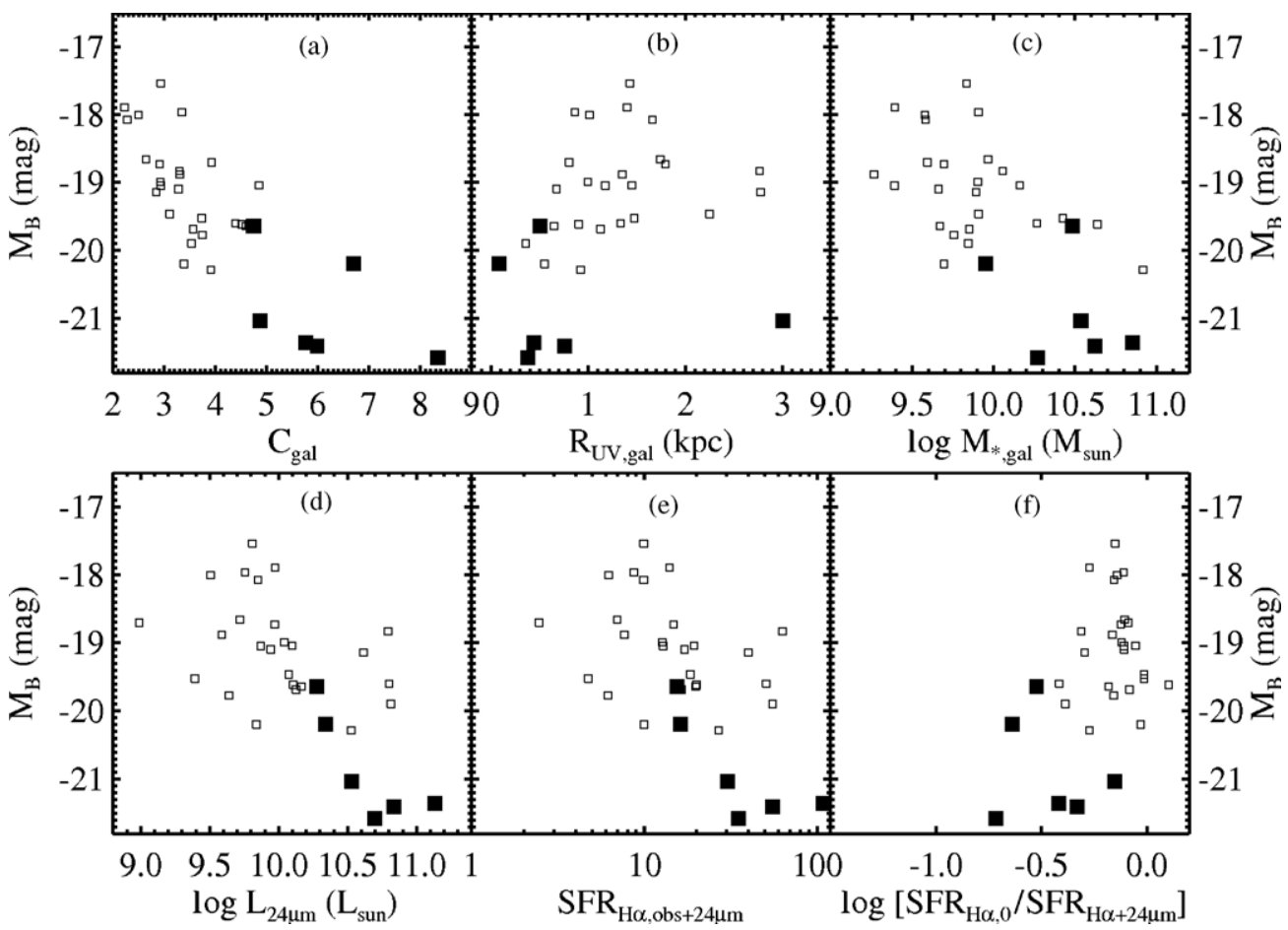

Figure 9. Correlations between the absolute $B$-band magnitudes $\left(M_{B}\right)$ of the star-forming clumps and various properties of the host galaxies measured from the $H S T$ optical image, the SDSS spectrum and the Spitzer IR photometry. The six DCOs are indicated by filled squares. Panels show (a) $M_{B}$ vs. optical concentration index, (b) galaxy UV half-light radius, (c) stellar mass, (d) $24 \mu \mathrm{m}$ luminosity, (e) total SFR, and (f) the ratio of the $\mathrm{H} \alpha \mathrm{SFR}, \mathrm{SFR} \mathrm{H} \alpha, 0$, corrected for dust using the Balmer lines to $\mathrm{SFR}_{\mathrm{H} \alpha, \mathrm{obs}+24 \mu \mathrm{m}}$, the SFR based on the attenuated $\mathrm{H} \alpha$ corrected for dust using the $24 \mu \mathrm{m}$ luminosity and Calzetti et al. (2007). We observe the following trends. The brightest clumps tend to have (a) larger host concentrations $C$, (b) smaller effective radii, (c) larger host stellar masses $M_{*}$, (d) larger host mid-IR luminosities, (e) larger host SFRs, and (f) relatively low total SFRs based on $\mathrm{H} \alpha$ corrected for dust using the Balmer decrement compared to SFRs based on $\mathrm{H} \alpha$ corrected for dust based on the $24 \mu \mathrm{m}$ emission from dust heated by young stars. The six DCOs are indicated by filled squares. The DCO having a relatively large UV half-light radius of $\sim 3 \mathrm{kpc}$ seen in panel (b) is object 080232. See Section 3.4 for details.

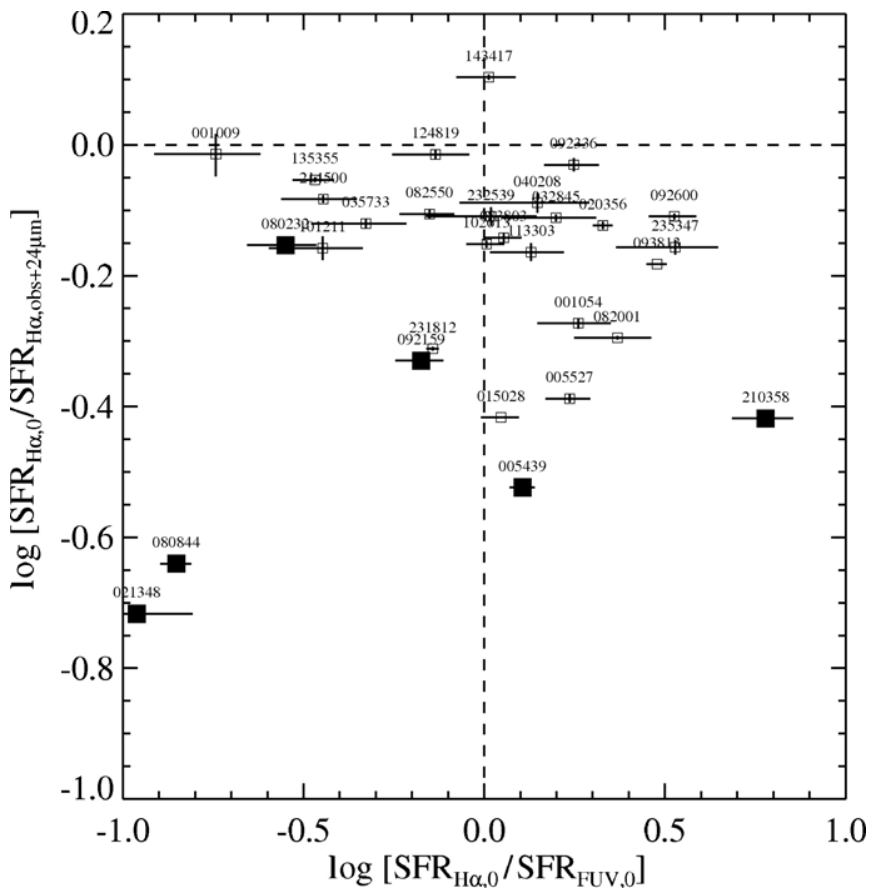

Figure 10. Ratio of $\mathrm{SFR}_{\mathrm{H} \alpha, 0} / \mathrm{SFR}_{\mathrm{FUV}, 0}$ vs. $\mathrm{SFR}_{\mathrm{H} \alpha, 0} / \mathrm{SFR}_{\mathrm{H} \alpha, \text { obs }+24 \mu \mathrm{m}}$ of clump host galaxies. The SFRs were calculated using the recipes given in Section 2.4 . Under reasonably standard assumptions for young starburst galaxies, all three SFR indicators are expected to give more or less consistent answers, and we expect the sources to line up on the dashed lines. We find significant outliers and discuss the implications in Sections 4.1 and 4.2. The DCOs are marked by filled squares.
(Figure 10), since AGNs are characterized by strong emission by warm dust peaking in the mid-IR (e.g., Spinoglio et al. 2002). It would not, however, explain the result in Figure 10 that the ratio of the extinction-corrected far-UV and $\mathrm{H} \alpha$ luminosity is also higher on average in the galaxies with DCOs than in typical star-forming galaxies, since in a Type $2 \mathrm{AGN}$ the continuum from the AGN itself will be very heavily obscured in the far-UV.

To further test this starburst/AGN composite hypothesis, we can use other emission-line diagnostic diagrams that are complementary to the BPT diagram. Following Veilleux \& Osterbrock (1987) and Kewley et al. (2006), we show in Figure 14 plots of $[\mathrm{S} \mathrm{II}] / \mathrm{H} \alpha$ versus $\left[\mathrm{O} \mathrm{III}^{\mathrm{II}} / \mathrm{H} \beta\right.$ and $[\mathrm{O} \mathrm{I}] / \mathrm{H} \alpha$ versus $[\mathrm{O} \mathrm{III}] / \mathrm{H} \beta$. Bonafide starburst-AGN composite systems will lie in the region intermediate between the locus of pure star-forming galaxies and pure AGNs in these plots. Instead, we see that the galaxies with the DCOs tend to lie in a kind of "no man's land" displaced from the locus of star-forming galaxies in a direction opposite to that of the AGN (they have weaker [S II] and [OI] emission than normal star-forming galaxies). Their anomalous behavior is even more dramatically illustrated in the right-most panel in Figure 14 where we plot [N II]/[S II] versus $[\mathrm{O} \mathrm{III}] / \mathrm{H} \beta$. As indicated in Figures 12 and 14 (small gray circles), similar behavior is seen in local IR-warm starbursts (Kewley et al. 2001).

Thus, the hypothesis that the compact clumps are found in starburst/AGN composite systems does not explain the properties of these objects. In future papers, we will describe observations with XMM-Newton, the Spitzer Infrared Spectrograph, and the European VLBI network that are designed to search for more direct signatures of the presence of an AGN. 

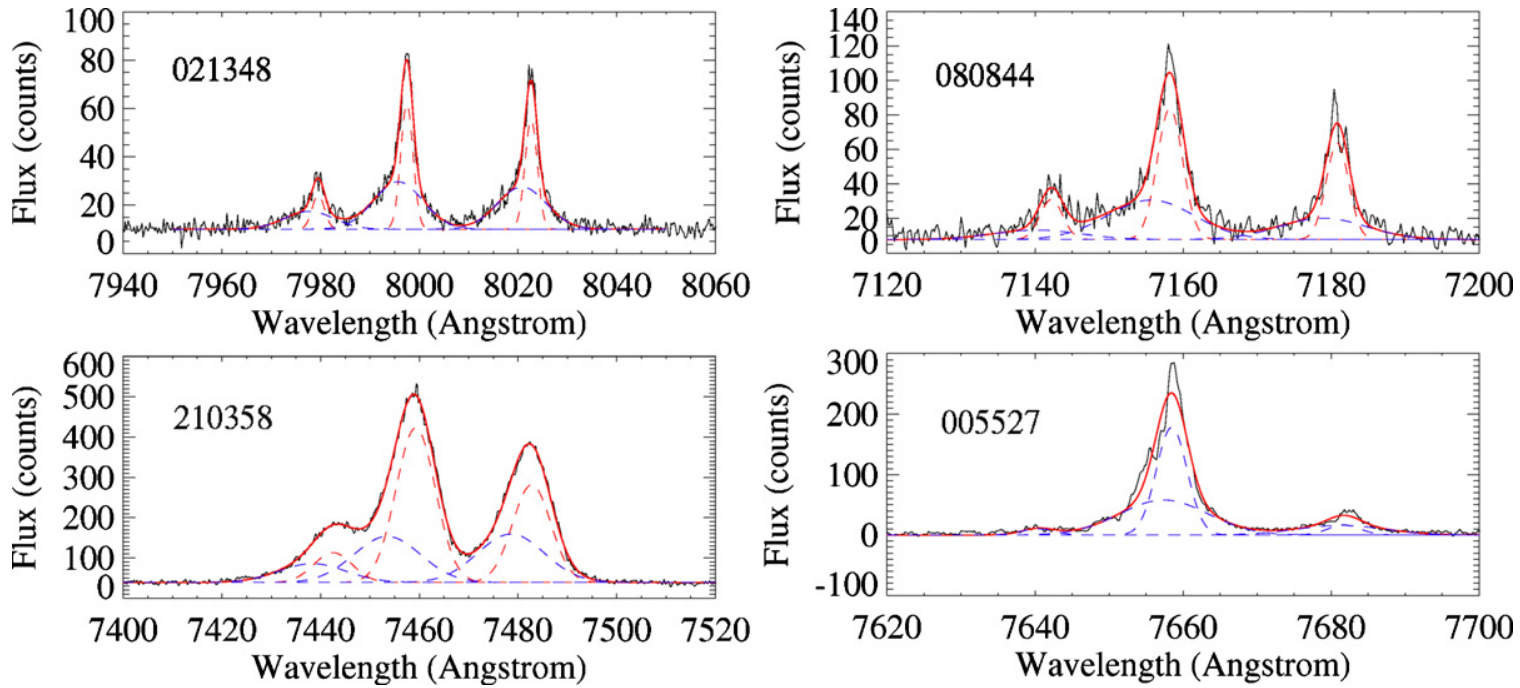

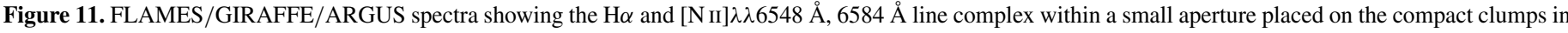

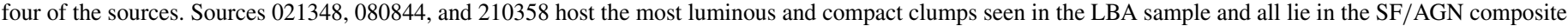

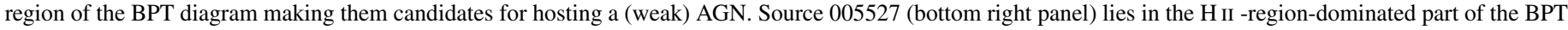

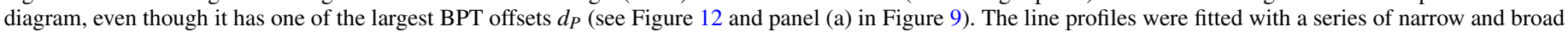

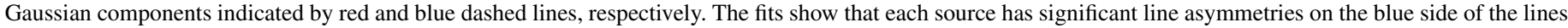

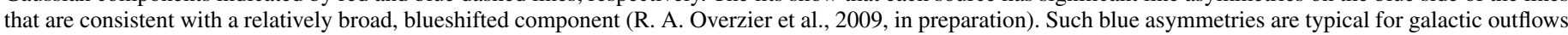
in starburst galaxies at both low and high redshift. See Section 4.1 for details.

(A color version of this figure is available in the online journal.)

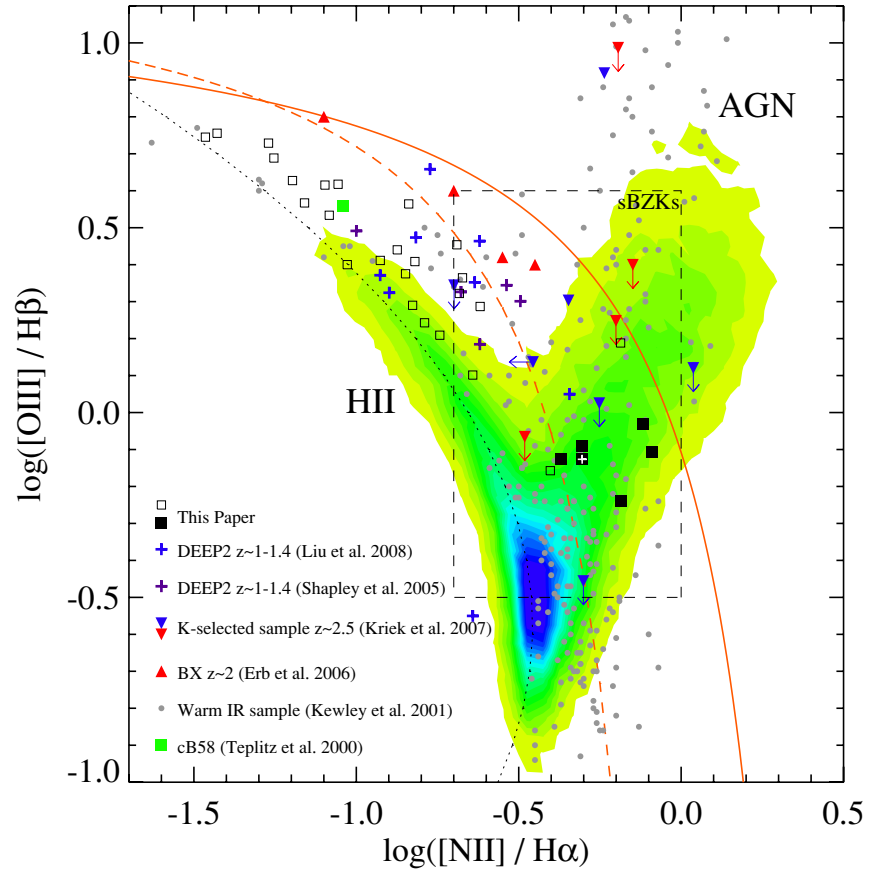

Figure 12. BPT diagram of the galaxies in our sample (open/filled squares). Filled squares correspond to the LBAs hosting the DCOs. Comparison data shown are samples of star-forming galaxies at high redshift (green square: LBG cB58, Teplitz et al. 2000; plus signs: DEEP2 galaxies at $z \sim 1$, Shapley et al. 2005; Liu et al. 2008; red triangles: "BX" galaxies at $z \sim 2$, Erb et al. 2006a; blue (red) upside-down triangles: star-forming (quiescent) $K$-selected galaxies at $z \sim 2.5$, Kriek et al. 2007; dashed box region: star-forming "s $B z K$ " galaxies at $z \sim 2$, Hayashi et al. 2009; Lehnert et al. 2009) and low redshift (gray circles: nearby sample of warm IR-luminous galaxies from Kewley et al. (2001). Contours show the density distribution of emission line galaxies in the SDSS. Lines show the boundary relations between star-forming galaxies and AGNs (Kauffmann et al. 2003, dashed line) and composite galaxies and AGNs (Kewley et al. 2006, solid line), and the SDSS star-forming ridge line from Brinchmann et al. (2008a). LBA 092159 (white cross) was found to contain a compact radio core using VLBI (in preparation). See Section 4.1 for details.

(A color version of this figure is available in the online journal.)

\subsection{Further Clues from the Emission-line Properties}

We have estimated the ages of the clumps and DCOs to be 5-100 Myr assuming a model of an instantaneous burst of star formation (see Table 3). We have also shown that there exist striking correlations between the properties of the clumps (e.g., luminosity), the properties of their host galaxies (e.g., mass, radius, and concentration), and the physical properties of the surrounding gas (e.g., ionization and density). The LBAs with DCOs are particularly extreme in these plots. Below we will discuss the most likely physical mechanisms that can explain these correlations.

The results presented in Section 3 showed that there are significant correlations between the properties of the massive compact clumps and the physical properties of the surrounding ionized gas as traced by the nebular emission-lines. In particular, the DCOs tend to be found in galaxies in which:

1. The SFR derived from the extinction-corrected $\mathrm{H} \alpha$ emission-line luminosity tends to be systematically smaller than that derived using the mid-IR and/or the extinctioncorrected far-UV continuum luminosities (see panel (f) in Figures 9 and 10). More properly, the ratio of the $\mathrm{H} \alpha$ luminosity to either the mid-IR or far-UV continuum is smaller on average than in typical star-forming galaxies.

2. The location of the galaxy in the diagnostic plot of [O III]/ $\mathrm{H} \beta$ versus [N II] $/ \mathrm{H} \alpha$ (BPT diagram, Figure 12) is more significantly displaced with respect to the locus of normal star-forming galaxies (see panels (a) and (b) in Figure 13). This displacement is in the sense of enhancements in one or both of the two above ratios.

3. The electron density in the ionized gas is significantly higher than in the other galaxies (panel (c) in Figure 13).

Occam's razor drives us to ask if there is a single explanation for all of these results. In Section 4.1, we have already argued that the contribution from an obscured, low-luminosity AGN could explain some but not all of the properties observed. Here 


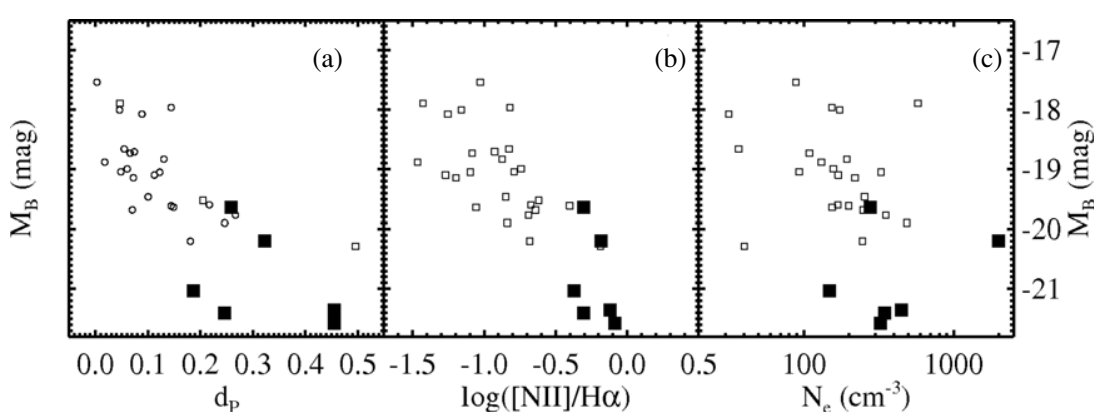

Figure 13. Correlations between the absolute $B$-band magnitudes $\left(M_{B}\right)$ of the star-forming clumps and various emission line properties of the host galaxies measured from the SDSS spectrum. In each plot, the six DCOs are indicated by filled squares. (a) $M_{B}$ vs. BPT diagram offset $d_{P},(\mathrm{~b}) \log ([\mathrm{N}$ II $] / \mathrm{H} \alpha)$, and (c) electron density $N_{e}$. The host galaxies of the brightest clumps tend to have larger BPT offsets $d_{P}$, larger $\log ([\mathrm{N}$ II $] / \mathrm{H} \alpha)$, and larger electron densities $N_{e}$. See Section 4.1 for details.
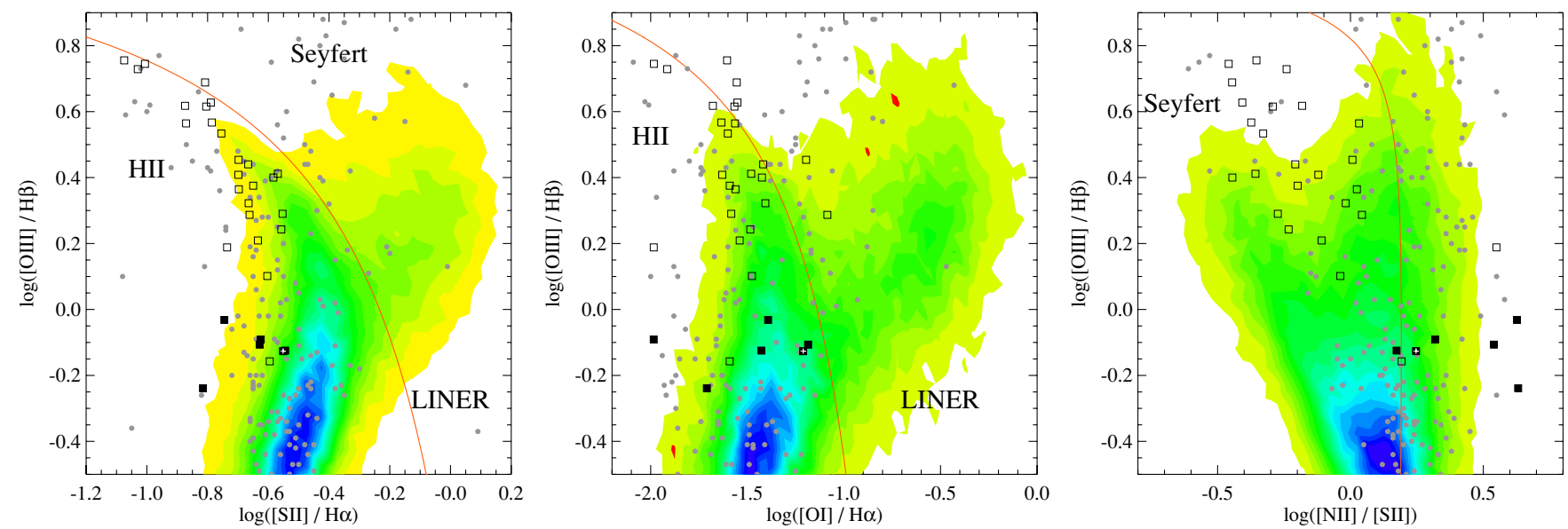

Figure 14. In order to further test the AGN hypothesis, we show plots of [S II] $/ \mathrm{H} \alpha$ vs. [O III]/H $\beta$ and [O I] $/ \mathrm{H} \alpha$ vs. [O III]/H $\beta$ (following Veilleux \& Osterbrock 1987; Kewley et al. 2006). Bona fide starburst-AGN composite systems will lie in the region intermediate between the locus of pure star-forming galaxies and pure AGNs in these plots. Instead, we see some of the galaxies with the more massive DCOs lie in a kind of "no man's land" displaced from the locus of star-forming galaxies in a direction opposite to that of the AGN (they have weaker [S II] and [OI] emission than normal star-forming galaxies). Their anomalous behavior is even more dramatically illustrated in the right-most panel where we plot $[\mathrm{N} \mathrm{II}] /[\mathrm{S} \mathrm{II}]$ vs. [O III]/H $\beta$. Gray circles indicate the nearby warm IR-luminous sample from Kewley et al. (2001). See the text in Section 4.1 for details.

(A color version of this figure is available in the online journal.)

we will consider two alternative possibilities: (1) the DCOs are short-duration starbursts caught at a time after the peak in the O-star population, and (2) the DCOs include "leaky" systems in which a large fraction of the Lyman continuum is escaping.

\subsubsection{The Aged Starburst Hypothesis}

The DCOs are extremely massive $\left(\sim 10^{9} M_{\odot}\right)$, compact $\left(\sim 10^{2} \mathrm{pc}\right)$ objects. These high densities $\left(\sim 10^{3} \mathrm{M}_{\odot} \mathrm{pc}^{-3}\right)$ imply very short dynamical times $(R / v \sim 1 \mathrm{Myr})$. On simple grounds of causality, it is therefore plausible that these objects could have formed all their stars in a Myr, a timescale significantly less than the characteristic lifetimes of the $\mathrm{O}$-stars that produce nearly all the ionizing radiation in a starburst. In such a case, the ratio of the ionizing luminosity to the non-ionizing far-UV luminosity changes rapidly with time, and falls dramatically for ages greater than about 5-6 Myr (e.g., Leitherer \& Heckman 1995). The dereddened UV/optical colors of the massive compact clumps shown in Figure 5 (while uncertain) are consistent with those of such "aged" instantaneous starbursts with ages of 1 to a few tens of Myr (see Table 3). This is consistent with BasuZych et al. (2007) who compared the SFRs derived from the UV, IR, and radio. We should note, however, that at least in one case (210358) we find evidence for WR stars in the SDSS spectrum indicating that its SFH must include a much more recent $(<5 \mathrm{Myr})$ burst.
If, however, the aged starburst hypothesis is correct for other objects, this would naturally explain the relative weakness of the $\mathrm{H} \alpha$ emission line in these galaxies. Such a model could also explain the peculiar location of these objects in the BPT diagram (Figure 12). For an instantaneous burst in the age range between about 10 and $50 \mathrm{Myr}$, the primary heating/ionizing source for the associated gas would be the mechanical energy supplied by supernovae rather than O-star-ionizing radiation (Leitherer \& Heckman 1995). Models of such shock-heated gas (e.g., Dopita \& Sutherland 1995; Allen et al. 2008) show an enhancement in the $[\mathrm{O}$ III $] / \mathrm{H} \beta$ and $[\mathrm{N} \mathrm{II}] / \mathrm{H} \alpha$ ratios, as we observe (Figure 12).

The expected supernova rate would be $\sim 4\left(M_{\text {clump }} / 10^{9} M_{\odot}\right)$ per decade. Such a high supernovae rate occurring in a very small region also naturally accounts for the high electron densities and thus the high gas pressures $\left(P / k \sim 10^{7} \mathrm{~K} \mathrm{~cm}^{-3}\right)$ in these objects. Following Chevalier \& Clegg (1985), in the absence of significant radiative losses, the central pressure inside a starburst region of radius $r_{100}$ (in units of $100 \mathrm{pc}$ ) with a supernova rate of $R_{\mathrm{SNe}}$ per decade is given by $P / k=1.4 \times 10^{8} R_{\mathrm{SNe}} r_{100}^{-2} \mathrm{~K}$ $\mathrm{cm}^{-3}$. Chevalier \& Clegg (1985) also demonstrate that this high-pressure gas will subsequently expand outward to form a galactic wind with a ram pressure that drops like $r^{-2}$. This wind would be sufficient to produce the observed gas pressures over $\mathrm{kpc}$ scales in the region surrounding the clump. The galactic wind will also accelerate emission-line clouds, leading to the 
broad blueshifted wings seen on the emission-line profiles in our objects (Figure 11). In fact, similar high gas pressures, broad blueshifted wings, and other signposts of galactic winds are common in intense local and high-redshift starbursts (e.g., Lehnert \& Heckman 1996; Lehnert et al. 2009; Shapiro et al. 2009).

This model fails however to explain the relative weakness of the $\left[\mathrm{S} \mathrm{II}_{\mathrm{II}}\right.$ and [O I] lines seen in Figure 14. Shock heating should lead to an enhancement in the relative strengths of these lines (see Figure 2 in Dopita \& Sutherland 1995). We are left with an otherwise attractive model that does not naturally explain all the properties of these objects.

\subsubsection{The Leaky Starburst Hypothesis}

An obvious way to lower the amount of observed $\mathrm{H} \alpha$ emission would be a "leaky" interstellar medium in which a significant fraction of the ionizing radiation produced by $\mathrm{O}$ stars escapes the galaxy. This hypothesis has a plausible physical basis: the feedback effect of concentrating the release of the kinetic energy from of-order $10^{7}$ supernovae $\left(\sim 10^{58} \mathrm{ergs}\right)$ all within the exceptionally small volume of a DCO may create large holes in the surrounding interstellar medium through which ionizing radiation could then escape. Taken at face value, Figure 10 would then imply that the majority of the ionizing radiation is escaping in our two most extreme objects (021348 and 080844). It is interesting to note that Shapley et al. (2006) find two out of 14 LBGs at $z \sim 3$ with a significant escape fraction ( $\left.f_{\text {esc }} \simeq 50 \%-100 \%\right)$ as measured from the ratio of the fluxes below and above the Lyman limit in UV spectra. Similarly, Iwata et al. (2009) report the detection of large $f_{\text {esc }}$ in 7 of 73 LBGs at $z \sim 3$. These fractions are similar to what is implied for our sample of LBAs under the leaky starburst hypothesis.

The leaky starburst hypothesis can readily account for the high electron densities (gas pressures) and the broad blue wings on the emission-line profiles: these are still intense starbursts and the same arguments as given above will apply. A leaky interstellar medium could also explain at least some aspects of the peculiar emission-line ratios we observe. Models of photoionized gas under "matter-bounded" conditions (e.g., Binette et al. 1996; Castellanos et al. 2002; Giammanco et al. 2005; Brinchmann et al. 2008a) show that the strengths of the $\left[\mathrm{OI}_{\mathrm{I}}\right.$ and $[\mathrm{S} \mathrm{II}]$ lines relative to $\mathrm{H} \alpha$ are decreased. This is because these lines are relatively strong in the warm partially neutral region lying beyond the edge of the Stromgren sphere, and this region is missing in matter-bounded conditions. These models show that the $[\mathrm{O} \mathrm{III}] / \mathrm{H} \beta$ ratio will also be enhanced in matter-bounded clouds, as we observe (Figure 12). However, this model fails to explain the enhanced $[\mathrm{N}$ II] $/ \mathrm{H} \alpha$ ratios seen for the DCOs in the BPT diagram. Models (B. Groves 2009, private communication) show that matter-bounded clouds in starbursts should have $[\mathrm{NII}] / \mathrm{H} \alpha$ ratios that are similar to or lower than ionization-bounded clouds.

A more fundamental objection to the leaky starburst hypothesis is that it is difficult to envisage a situation in which a sizable fraction of the ionizing radiation escapes the galaxy, but the majority of the non-ionizing UV radiation is absorbed by dust inside the galaxy (as is required by the high mid-IR luminosities we see). For this reason, we regard the "aged starburst" hypothesis as the more likely one.

In the future, we will use long-slit and integral field spectroscopy in order to spatially resolve and disentangle the line emission coming from the clumpy versus the diffuse regions seen in the HST images.

\section{DISCUSSION OF THE STRUCTURAL PROPERTIES OF LBAs}

The peculiar emission-line properties discussed above show the presence of significant feedback processes between the massive star-forming regions in LBAs and their surrounding ISM. Here, we will focus on the remarkable stellar properties of the massive clumps and the DCOs in particular, by asking the question to what kind of structural components of galaxies do they correspond?

The DCOs are only marginally resolved in the HST UV images, with implied radii of no greater than $\sim 100 \mathrm{pc}$ (see Figure 7). The fainter clumps are typically several times larger than this. The masses of the DCOs estimated in Section 3 range from a few $\times 10^{8}$ to a few $\times 10^{9} M_{\odot}$, while the more typical clumps have masses in the range of a few $\times 10^{7}$ to a few $\times 10^{8} M_{\odot}$. This implies very high stellar mass surface densities. For the typical clumps values for the effective surface mass density $\left(\Sigma_{e} \approx M_{* \text {, clump }} /\left(2 \pi R_{e}^{2}\right)\right)$ range from $\sim 10^{8}$ to $10^{9} M_{\odot}$ $\mathrm{kpc}^{-2}$, while for the DCOs $\Sigma_{e} \sim 10^{9.5}$ to $10^{11} M_{\odot} \mathrm{kpc}^{-2}$. What then will the clumps and DCOs evolve into? Can we identify such evolved counterparts in local galaxies?

\subsection{Super Star Clusters and Central Massive Objects?}

Massive clusters are observed in and around the nuclei of many nearby late- and early-type galaxies, mergers and merger remnants, and AGNs (e.g., Carollo et al. 1997; Geha et al. 2002; Böker et al. 2004; Maraston et al. 2004; Walcher et al. 2005; Seth et al. 2008). The largest stellar clusters typically have sizes of only a few pc (the largest being $\approx 10 \mathrm{pc}$ ) and masses rarely exceeding $\simeq 10^{7} M_{\odot}$. Their structural properties are thus similar to those of massive globular clusters in the Milky Way and local galaxies, and also to the young "super star clusters" (SSCs) found in starburst galaxies (Meurer et al. 1995).

The most luminous star clusters in normal and starburst galaxies lie along a relation between the absolute optical magnitude of the cluster and the global SFR of the host galaxy (Larsen 2002), a relation that is mainly driven by the relation between the luminosity of the brightest cluster and the total number of clusters formed in a galaxy (Whitmore et al. 2007). The correlation implies that for galaxies having SFRs as high as LBAs even the brightest clusters should only have $M_{V} \simeq-16$ to $-14\left(M_{V}=-1.87 \log (\mathrm{SFR})-12.14\right.$; see Weidner et al. 2004) compared to $-21.5 \lesssim M_{V} \lesssim-18$ found in Figure 5 . This discrepancy could be partly reconciled given that the LBA clusters are systematically younger than the brightest clusters observed in normal galaxies, since a star cluster fades by $\sim 3$ mag in the first few 100 Myr. However, this scenario still largely falls short for any of the luminous clumps/DCOs having $-21.5<M_{V}<-19$.

The most massive cluster-like object known to date, object W3 in the merger remnant NGC 7252, has a mass of $(8 \pm$ 2) $10^{7} M_{\odot}$ and a radius of $17.5 \pm 1.8 \mathrm{pc}$ (Maraston et al. 2004). Interestingly, its high mass and small size are very much at odds with any kind of stellar cluster found to date. Curiously, the properties of object W3 are perhaps more similar to that of ultra-compact dwarf galaxies (e.g., Mieske et al. 2004). If we extrapolate from its current luminosity at an age of $\sim 400 \mathrm{Myr}$ to that expected at $10 \mathrm{Myr}$, we find $M_{V}^{10 \mathrm{Myr}} \simeq-19.2$ (Bastian 2008), still over 2 mag fainter than some of the DCOs.

Although the stellar mass surface densities that we derive $\left(\sim 10^{8.5}-10^{11} M_{\odot} \mathrm{kpc}^{-2}\right.$ ) are comparable to that of typical massive clusters mentioned above, the sizes are about an order of 
magnitude larger $(\sim 100 \mathrm{pc})$, and the masses are up to two orders of magnitude larger $\left(\lesssim 10^{9} M_{\odot}\right)$. Thus, the massive clumps and DCOs identified in the LBA sample appear as significantly scaled-up versions of known types of massive star clusters, and are not simply an extension of the cluster luminosity function as derived from nearby star-forming galaxies.

Furthermore, nuclear super star clusters and supermassive black holes are jointly referred to as central massive objects (CMOs). CMOs follow a tight correlation with the total mass of the galaxy over many orders of magnitude in stellar mass. The central star clusters in low luminosity early-types and spiral bulges, as well as the masses of the central supermassive black holes in $L \geqslant L_{*}$ early-types all lie on the relation $M_{\mathrm{CMO}} \approx 0.002 M_{\text {gal }}$ with small scatter (e.g., Magorrian et al. 1998; Marconi \& Hunt 2003; Côté et al. 2006; Ferrarese et al. 2006; Wehner \& Harris 2006; Rossa et al. 2006). Simulations of both isolated and merging galaxies reproduce the observed correlation well (e.g., Li et al. 2007). As we discuss below in more detail, the clumps and DCOs typically represent several percent of the total stellar mass of the surrounding galaxy. These mass fractions are therefore over an order of magnitude higher than in the CMOs.

Could the massive clumps or DCOs consist of multiple SSCs? If so, how long would it take this ensemble to merge into a single object? To address this, we assume that a clump of mass $10^{8} M_{\odot}$ actually consists of $10-100$ SSCs each having a mass of $10^{6-7} M_{\odot}$ distributed over a region with a $100 \mathrm{pc}$ radius within which the velocity dispersion $\sigma_{\text {clump }} \approx \sqrt{G M_{\text {clump }} / 5 R_{e}} \approx$ $30 \mathrm{~km} \mathrm{~s}^{-1}$. Then the timescale over which a single SSC migrates inward as a result of dynamical friction is on the order of only 10-20 Myr (Binney \& Tremaine 2008), comparable to the typical ages we have derived for the clumps. This suggests that a given massive clump (or DCO) is likely to have evolved into a single very massive object, even if it started out as an ensemble of SSCs.

We conclude that the clumps and DCOs are very different structurally from typical nuclear (super) star clusters.

\subsection{Extra Light and Central "Cusps" in Elliptical Progenitors?}

For the discussion below, it is important to point out that the DCOs (and, more generally, the most luminous clumps) tend to appear in the more massive galaxies in our LBA sample (see panel $\mathrm{c}$ in Figure 9). The DCOs also each sit in the middle of extended, perturbed disk-like structures (hence the correlation between clump luminosity and the optical concentration index, panel a of Figure 9). Another characteristic (by definition) is that the DCOs are single objects rather than the multiple (fainter) clumps as seen in many of the other objects.

In Figure 15, we plot the clump mass $\left(M_{*}\right)$ and effective radius $\left(R_{e}\right)$ versus the effective mass density $\left(\Sigma_{e}\right)$ for the brightest clump in each LBA, and compare with a collection of results from the literature. The comparison data shown were extracted from a compilation of literature results presented in Figure 45 in Hopkins et al. (2009), showing globular clusters (crosses), nuclear star clusters (triangles), and the extra-light components in early-type galaxies from the samples of Kormendy et al. (2009, stars) and Lauer et al. (2007, circles). The high masses of $\sim 10^{9} M_{\odot}$ derived for the DCOs combined with the strong constraints on their small effective radii $(\sim 100 \mathrm{pc}$ as evidenced by the HST diffraction spikes in Figure 4) yield the highest mass surface densities found in our sample, $\Sigma_{M} \approx 10^{10}$ $10^{11} M_{\odot} \mathrm{kpc}^{-2}$ (see Table 4).
Hopkins et al. (2009) show that this region of parameter space in the $M_{*}$ and $R_{e}$ versus $\Sigma_{e}$ planes is almost exclusively occupied by the so-called central excess or "extra-light" component seen in local elliptical galaxies (stars and circles in Figure 15; Crane et al. 1993; Lauer et al. 1995; Kormendy et al. 2009, and references therein). This extra light is characterized as an inner $(\lesssim 1 \mathrm{kpc})$ component that rises more steeply than expected from the extrapolated Sersic power law that fits the outer profile of ellipticals well. The so-called "cusps" have effective radii of $\sim 50-500 \mathrm{pc}$ and show a trend of decreasing density with increasing mass and in that sense they lie on the same sequence formed by elliptical galaxies. The cuspy cores are furthermore typical for relatively low-mass $\left(\leqslant L_{*}\right)$ ellipticals, while they are relatively uncommon for the most massive ellipticals. Kormendy et al. (2009) suggest that the core-cusp dichotomy in local ellipticals may correspond to their formation history: the extralight or cusp components are very likely the remnant signature of a massive central starburst in a dissipative ("wet") merger, while the extended cores in core-dominated ellipticals are consistent with being the relaxed descendants of dissipationless ("dry") mergers (e.g., Graham 2004; Shankar \& Bernardi 2009).

While none of the clumps bear any structural similarities with both globular and nuclear star clusters (crosses and triangles), the DCOs in the $M_{*}, R_{e}, \Sigma_{e}$ plane appear structurally similar to the extra-light components in early-type galaxies (stars and circles). Hopkins et al. (2009) investigate in detail how the central mass associated with the extra-light component is related to the remnant of a massive central starburst fueled by dissipative merging, and use extensive simulations to show that such a mechanism successfully reproduces the observed profiles of elliptical galaxies (Hopkins et al. 2008, 2009). In particular, they find a strong correlation between the galaxy stellar mass and the fraction of the total stellar mass that is in the extra-light component $\left(f_{\text {extra }}\right)$, as well as a one-to-one correlation between the extra-light mass fraction and the fraction of the total stellar mass that formed in the dissipative starburst $\left(f_{\text {starburst }}\right)$, with roughly a factor of $\sim 2$ intrinsic scatter in $f_{\text {starburst }}$ and a factor of $\sim 4$ scatter in $f_{\text {extra }}$ at each $M_{*}$. For stellar masses in the range of $10^{10}-10^{11} M_{\odot}$ the $f_{\text {starburst }}\left(f_{\text {extra }}\right)$ ranges from a few to $50 \%$, consistent with the extra-light fractions measured in real ellipticals by Hopkins et al. (2009).

We can compare this with the ratio of the central clump mass to the total stellar mass we found for the DCOs. Due to the uncertainties in the mass estimates involved, we estimate the DCO mass by taking the average of the values derived using single burst and continuous SFHs. The result is shown in Figure 16. We find mass fractions typically of a few percent ( $f_{\text {DCO }} \sim 2 \%-4 \%$ ), object 021348 being a notable exception ( $f_{\text {DCO }} \sim 23 \%$, see also Table 4 ). Except for object 021348 , which agrees well with the cuspy cores, the mass fraction of the DCOs appears to be relatively low compared to the early-type cusps plotted in Figure 16 (stars and circles). However, the mass fractions we find are still significant and not inconsistent with the data.

Alternatively, we should note that the special region of parameter space in $M_{*}, R_{e}$ and $\Sigma_{e}$ occupied by both the DCOs and the cuspy cores appears to represent a particular transition region between massive, cluster-sized objects on one hand and entire galaxies on the other. While low-mass star clusters follow the scaling relations of globular clusters for masses $\lesssim 10^{6} M_{\odot}$, a wide variety of higher mass objects ranging from the most massive nuclear star clusters, dwarf-galaxy transition objects, ultracompact dwarf galaxies, dwarf elliptical nuclei, 


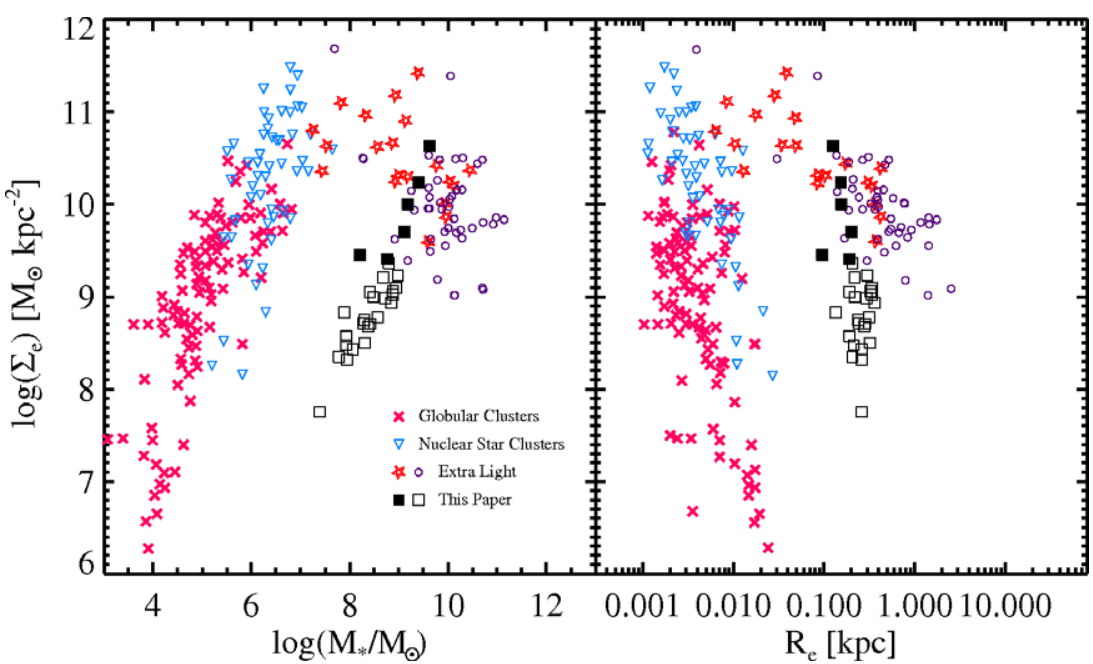

Figure 15. Mass $\left(M_{*}\right)$ and effective radius $\left(R_{e}\right)$ vs. the effective mass surface density $\left(\Sigma_{e}\right)$ of the brightest clump identified in each galaxy (squares). The comparison data shown were extracted from a compilation of literature results presented in Figure 45 in Hopkins et al. (2009), showing globular clusters (crosses), nuclear star clusters (triangles), and the extra-light components in early-type galaxies from the samples of Kormendy et al. (2009, stars) and Lauer et al. (2007, circles) as measured by Hopkins et al. (2009). Objects that we identify as DCOs based on their location in the $M_{*}, R_{e}, \Sigma_{e}$ plane in combination with the large total stellar masses and concentrations of their host galaxies are indicated with filled squares. We suggest that DCOs could be the progenitor starbursts of the central "extra-light" component in local cuspy elliptical galaxies. See Section 5.2 for details.

(A color version of this figure is available in the online journal.)

Table 4

Properties of the Massive, Unresolved Clumps ("DCOs") Discussed in Section 5

\begin{tabular}{ccccccccccc}
\hline \hline $\mathrm{ID}^{\mathrm{a}}$ & $f_{\mathrm{UV}}{ }^{\mathrm{b}}$ & $f_{\mathrm{OPT}}{ }^{\mathrm{b}}$ & $\begin{array}{c}\log \left\langle M_{*, \mathrm{clump}}\right\rangle^{\mathrm{c}} \\
\left(M_{\odot}\right)\end{array}$ & $\begin{array}{r}R_{e}{ }^{\mathrm{d}} \\
(\mathrm{pc})\end{array}$ & $\begin{array}{c}\log \Sigma_{M}{ }^{\mathrm{e}} \\
\left(M_{\odot} \mathrm{kpc}^{-2}\right)\end{array}$ & $\begin{array}{c}\sigma_{v}{ }^{\mathrm{f}} \\
\left(\mathrm{km} \mathrm{s}^{-1}\right)\end{array}$ & $\begin{array}{c}t_{\mathrm{dyn}}{ }^{\mathrm{g}} \\
(\mathrm{Myr})\end{array}$ & $\begin{array}{c}t_{\text {fric }} \mathrm{h} \\
(\mathrm{Myr})\end{array}$ & $\begin{array}{c}\log \left\langle M_{*, \mathrm{gal}}\right\rangle^{\mathrm{i}} \\
\left(M_{\odot}\right)\end{array}$ & $\left\langle M_{\text {clump }}\right\rangle /\left\langle M_{*, \mathrm{gal}}\right\rangle^{\mathrm{j}}$ \\
\hline 210358 & 0.48 & 0.20 & 9.41 & 153 & 10.24 & 120 & 1 & 65 & 10.9 & 0.04 \\
021348 & 0.49 & 0.36 & 9.63 & 125 & 10.64 & 171 & 1 & 77 & 10.3 & 0.23 \\
092159 & 0.46 & 0.29 & 9.11 & 202 & 9.70 & 74 & 3 & 50 & 10.6 & 0.03 \\
080232 & 0.20 & 0.17 & 9.18 & 156 & 10.00 & 91 & 2 & 153 & 10.5 & 0.04 \\
080844 & 0.77 & 0.30 & 8.21 & 95 & 9.46 & 38 & 2 & 56 & 10.0 & 0.02 \\
005439 & 0.43 & 0.23 & 8.77 & 190 & 9.41 & 52 & 4 & 70 & 10.5 & 0.02 \\
\hline
\end{tabular}

Notes.

${ }^{a}$ ID number of the host galaxy of the DCO.

${ }^{\mathrm{b}}$ Fractions of DCO-to-total light measured in the UV $\left(f_{\mathrm{UV}}\right)$ and optical $\left(f_{\mathrm{OPT}}\right)$. A circular aperture of 0 '. 2 radius was used for the DCO, and an aperture of $10^{\prime \prime}$ radius was used to estimate the total light. Companion galaxies were masked out and a large object-free annulus near the source was used for background subtraction. For object 080844, the UV/optical light fractions correspond to those measured in the filters F330W/F850LP, instead of F150LP/F606W. The total optical light for 080844 was measured within an aperture of $5^{\prime \prime}$.

${ }^{c}$ Average of the single burst and continuous SFHs clump masses of Table 3.

${ }^{\mathrm{d}}$ Physical optical half-light radius measured within the 0". 4 diameter aperture used in Section 3.3.

${ }^{\text {e }}$ Stellar mass surface density $\Sigma_{M}=\left\langle M_{*, \text { clump }}\right\rangle / 2 \pi R_{\text {clump }}^{2}$.

${ }^{\mathrm{f}}$ Clump velocity dispersion assuming spherical geometry $\sigma_{v, \text { clump }}=\sqrt{\frac{G\left\langle M_{*, \text { clump }}\right\rangle}{5 R_{\text {clump }}}}$.

${ }^{\mathrm{g}}$ Dynamical time $t_{\mathrm{dyn}}=R_{\text {clump }} / \sigma_{v \text {, clump }}$

${ }^{\mathrm{h}}$ Dynamical friction time $t_{\text {fric }}=\left(\frac{2.64 \times 10^{11}}{\frac{1}{2} \ln \left(1+\Lambda^{2}\right)}\right)\left(\frac{R_{\mathrm{gal}}}{2 \mathrm{kpc}}\right)^{2}\left(\frac{\sigma_{v, \text { gal }}}{250 \mathrm{~km} \mathrm{~s}^{-1}}\right)\left(\frac{10^{6} M_{\odot}}{\left\langle M_{* \text {,clump }}\right\rangle}\right)$, where $\Lambda=\frac{R_{\mathrm{gal}} \sigma_{v, \text { gal }}^{2}}{G\left\langle M_{* \text {,clump }}\right\rangle}$.

${ }^{\mathrm{i}}$ Galaxy stellar mass estimated from the average of the SED and dynamical masses.

j Stellar mass fraction of the clumps.

and the early-type cusps themselves all appear to lie on the same scaling relations for spheroidal galaxies (e.g., KisslerPatig et al. 2006; Hopkins et al. 2009). Although this overlap may be partially coincidental, perhaps it can be explained if these objects are all the product of some generic dissipational process.

In contrast with the DCOs, the much less extreme clumps indicated in Figure 15 are typically found in LBAs containing multiple clumps distributed within their central regions (see Figures 1 and 3). For a clump of mass $M_{\text {clump }} \sim 10^{8} M_{\odot}$ moving within a disk of mass $M_{\text {disk }} \sim 10^{10} M_{\odot}$ and a disk radius of $R_{\text {disk }} \sim 1.5 \mathrm{kpc}$ (the average optical half-light radius) we find $\sigma_{\text {disk }} \approx \sqrt{G M_{\text {disk }} / 3.4 R_{\text {disk }}} \approx 80 \mathrm{~km} \mathrm{~s}^{-1}$ and a dynamical friction time of $\sim 100-200 \mathrm{Myr}$. This is several times longer than the ages that we have derived for the individual clumps. Thus it is plausible that at this relatively early dynamical stage we usually see several such clumps within the effective radius of the galaxy. The diffuse light seen in the HST images may build up as the clumps lose stars due to tidal stripping while they interact with each other and with the disk and slowly migrate inward. This process may eventually lead to the formation of an inner bulge as well as the Sersic profile typical of disk galaxies (Noguchi 


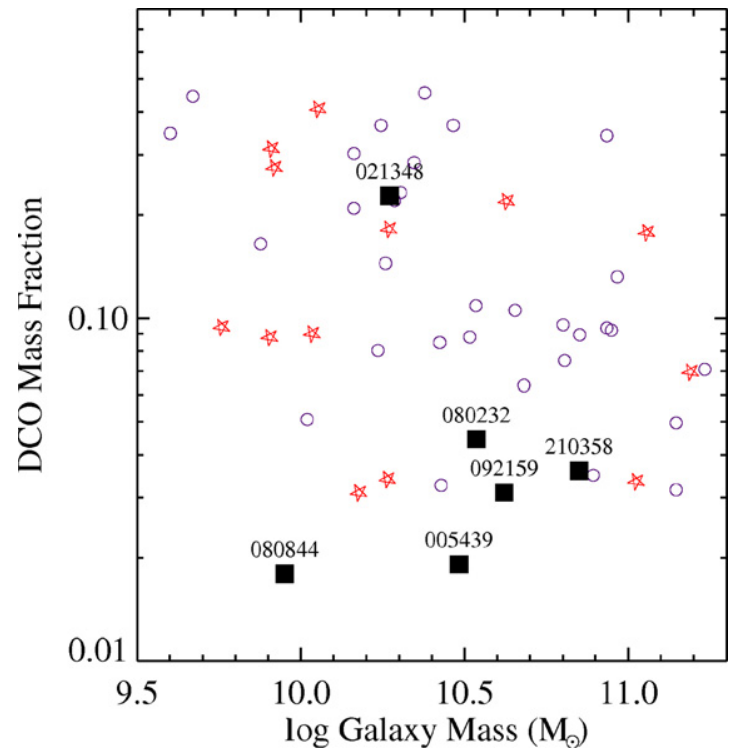

Figure 16. Fraction of the stellar mass in the DCOs (see Figure 4) vs. the total stellar mass of the six objects. The DCOs contain $2 \%-23 \%$ of the total stellar mass, suggesting that they could be the hypothesized progenitor starbursts related to the central "extra-light" component observed in local cuspy elliptical galaxies. This is also consistent with their total stellar masses of $10^{10} \lesssim M_{*} / M_{\odot} \lesssim 10^{11}$. The comparison data shown (stars and circles; taken from Figure 20 in Hopkins et al. 2009) correspond to the same extra-light components as plotted in Figure 15. See Section 5.2 for details.

1999; Immeli et al. 2004; Carollo et al. 1997; Bournaud et al. 2007, 2008; Elmegreen et al. 2008a, 2008b, 2009).

\subsection{Relation to Mergers and IR-luminous Galaxies}

Although most LBAs were unresolved in the original SDSS images, some showed evidence for close companions or faint extended emission. One of the major results from the HST images is that most of them show very complex morphologies at scales well below the SDSS seeing or sensitivity. We interpret the large array of morphologies in Figure 1 as evidence that most LBAs are undergoing or have undergone an interaction or merger. ${ }^{15}$ We also see a small number of objects that do not show unambiguous evidence of merging such as tidal tails or companions, but that are nonetheless often highly peculiar as evidenced by ring or chain-like morphologies (e.g., object 102613 and objects $082001,082550,083803$ ). These observations suggest that the starbursts in LBAs and the formation of the massive stellar clumps are somehow linked to the mergers (e.g., Larson \& Tinsley 1978; Lambas et al. 2003; Nikolic et al. 2004; Woods et al. 2006; Owers et al. 2007; Li et al. 2008). The LBAs must also be sufficiently gas-rich in order for any starburst trigger to be effective. Simulations show that the inflow of gas ensures that the pressure in the ISM becomes larger than the internal pressure of giant molecular clouds, and that the clouds can collapse and form massive clusters before they are disrupted by supernova explosions (Bekki 2004).

It is important to note that the spectral energy distributions of LBAs are very different from typical starburst galaxies in the local universe. These are predominantly associated with the highly dust-obscured LIRGs $\left(10^{11} \leqslant L_{\mathrm{IR}} \leqslant 10^{12} L_{\odot}\right)$ and ULIRGs $\left(L_{\mathrm{IR}}>10^{12} L_{\odot}\right)$. At least ULIRGs are known

\footnotetext{
15 Here we use the term "merger" in the widest sense of the word, i.e., it is not limited to "major mergers" but includes minor mergers and other mechanisms that may deliver a supply of gas-rich material.
}

to be strongly associated with the different phases of galaxygalaxy mergers, with about equal fractions showing dual nuclei (pre-merger) and showing indication of post-merging (after the separate nuclei have coalesced and a period of violent relaxation starts forming an extended spheroidal component) (e.g., Sanders \& Mirabel 1996; Genzel et al. 2001; Tacconi et al. 2002; Dasyra et al. 2006a, 2006b; Arribas et al. 2008). These mergers are found to be limited to galaxy pairs having near-equal mass ratios (1.5:1 on average, with a maximum of $3: 1)$. The less luminous LIRGs are typically associated with much less violent events that are less effective at triggering a major starburst phase as observed in ULIRGs, perhaps as a result of smaller gas fractions, orbital parameters, and larger pair mass ratios (or a combination thereof). As shown in Arribas et al. (2008), about one-third of LIRGs appear in single isolated (spiral) galaxies. While none of the LBAs qualify as ULIRGs, there is some overlap with the LIRGs since $~ 30 \%-50 \%$ of LBAs have $L_{I R}>10^{11} L_{\odot}$. As such, LBAs likely represent a population of relatively unobscured mergers or merger remnants that share some characteristics with LIRGs. The number density of LBAs is much lower than that of LIRGs. If the two populations are somehow connected, the LBAs could represent a very short evolutionary phase during which the starburst can be seen relatively unobscured. Alternatively, or in addition, LBAs might be seen at a special viewing angle, perhaps along the axis where the starburst has blown a hole in the ISM and is leaking Lyman continuum photons.

\subsection{Implications for Black Hole Formation}

We have shown that the LBAs are consistent with galaxies that are in the very early stages of forming their central bulge and/or core excess. Local galaxies have tight correlations between the mass of their central black holes and that of their bulge and total mass. It is thus interesting to ask whether there is any evidence that a large black hole mass is accumulating in the LBAs as well. The DCOs are particularly intriguing in this regard: such very massive and compact objects appear to be the ideal breeding grounds for a supermassive black hole. As we have no means of directly detecting the gravitational signature of a black hole inside the massive clumps, the only way to infer the presence of a massive black hole would be through the spectral signature of an actively accreting AGN. As discussed in Section 4.1, we did not find any direct evidence for energetically significant AGNs in the DCOs.

It has long been suggested that the mass loss from stars and supernovae in a very massive compact cluster at the center of a galaxy may play a major role in the fueling of a supermassive black holes (Norman \& Scoville 1988). While the stellar winds from $\mathrm{O}$ and WR stars and the ejecta from core-collapse supernovae may in principle provide a significant amount of material available for accretion during the early stage of a massive central starburst, these are not expected to be viable fueling mechanisms because the speed of the outflowing stellar ejecta is far in excess of the escape velocity from the star cluster (Norman \& Scoville 1988). Norman \& Scoville (1988) argue that the high mass-loss rates and the low mass-loss speeds of the winds from evolved AGB stars may offer a steady flow of material that will be retained within the potential well of the star cluster. It can then cool and (some fraction) can be accreted by the supermassive black hole. Thus, the growth phase of the supermassive black hole (a powerful AGN) is expected to occur only after the time that the mechanical feedback energy input from core collapse supernovae has subsided and the progenitors 
of AGB stars have reached the end of their main sequence lifetimes. This will occur at $\sim 50 \mathrm{Myr}$ after the onset of the starburst (Leitherer \& Heckman 1995), and this age constraint is consistent with the observed ages of the nuclear starbursts observed in a sample of a local AGN (Davies et al. 2007). Under the assumption of an instantaneous burst, the ages of the DCOs shown in Figure 4 and listed in Table 4 are generally much lower (5-29 Myr) than $50 \mathrm{Myr}$. Thus, it is perhaps not unexpected that under such a scenario the DCOs are currently not capable of efficiently fuelling a supermassive black hole.

We also note that in the "migrating-clump model" advocated by Bournaud et al. (2007, 2008) and Elmegreen et al. (2008b), supermassive nuclear black holes may form under the assumption that each of the clumps gave rise to an intermediate mass black hole prior to its migration toward the center where they can coalesce. Such a mechanism may be able to explain the presence of seed black holes of substantial mass in the centers of gas-rich galaxies dominated by massive, turbulence-induced star-forming clumps. Both mechanisms described above may therefore predict significant delay times between the onset of a starburst and that of the AGN.

\section{SUMMARY AND CONCLUDING REMARKS}

We have presented the results of the analysis of a sample of 30 low-redshift $(z<0.3)$ galaxies selected from the union of the GALEX and SDSS surveys to have the high far-UV luminosities and small sizes characteristic of the high-redshift LBGs (Heckman et al. 2005; Hoopes et al. 2007; Basu-Zych et al. 2007; Overzier et al. 2008). These "LBAs" offer the opportunity of investigating the physical processes occurring in LBGs in considerably more detail than is possible at high redshift. ${ }^{16}$ In this paper, we reported on the internal structures of the LBA's using HST optical and UV images. We complemented these data with mid/far-IR photometry and optical spectroscopy from the SDSS and the VLT.

We have shown that the morphologies of the LBAs are dominated by massive compact clumps of young stars. Our most unexpected and potentially important result is that we have found six galaxies in which the galaxy UV morphology is dominated by a single very compact object which is unresolved or only marginally resolved in the HST images. These DCOs have estimated masses of few $\times 10^{8}$ to few $\times 10^{9} M_{\odot}$ and radii of-order $100 \mathrm{pc}$ or less. In the other LBAs, we instead find multiple clumps per galaxy, the brightest of which have estimated masses of typically a few $\times 10^{8} M_{\odot}$ and radii of several hundred pc.

We show that the galaxies containing the DCOs differ from the other LBAs in many respects.

1. Their host galaxies tend to be more massive than the other LBAs (median masses of $\sim 3 \times 10^{10}$ versus $\sim 6 \times 10^{9} M_{\odot}$, respectively).

2. They have systematically higher central concentrations of light than the other LBAs (the DCO is typically located near the center of a larger disk or envelope).

\footnotetext{
16 Heckman et al. (2005) coined the term "living fossils" in relation to the Lyman break analogs sample then discovered. Indeed, an apt analogy in the field of evolutionary biology is presented by the Coelacanth. A modern-day analog of this species of fish, known from the fossil record and believed to be extinct since the end of the Cretaceous period, was discovered in 1938. The find illustrates the use of analogs in the study of cosmology. Even though the local analogs will differ from their distant look-a-like to some level of detail, and even though their local environment has probably changed drastically, they offer an entirely new way of investigating the fossil record alongside with other, more traditional lines of research.
}

3. The DCOs are found in galaxies with high mid-infrared luminosities: $L_{24 \mu \mathrm{m}} \sim 10^{10.3}$ to $\sim 10^{11.2} L_{\odot}$, roughly an order of magnitude more luminous than the other LBAs and comparable to luminous infrared galaxies.

4. The galaxies with a DCO are more strongly displaced from the locus of normal star-forming galaxies in the standard BPT diagnostic emission-line ratio diagram. This displacement is in the sense of having larger $[\mathrm{O} \mathrm{III}] / \mathrm{H} \beta$ and/or larger $[\mathrm{N} \mathrm{II}] / \mathrm{H} \alpha$ ratios.

5. The DCOs tend to occur in galaxies with higher gas densities and pressures as traced by the ionized gas $(P / k \sim$ $10^{7} \mathrm{~cm}^{-3} \mathrm{~K}$ ).

6. The DCOs are found in galaxies in which the SFR inferred from the dust-corrected $\mathrm{H} \alpha$ luminosity tends to be smaller than that derived from the mid-IR luminosity and/or the extinction-corrected far-UV luminosity. In the most extreme cases, this discrepancy reaches roughly an order of magnitude.

We have shown that the DCOs are not Type 1 (unobscured) AGNs. We find no evidence for the presence of a very broad (several thousand $\mathrm{km} \mathrm{s}^{-1}$ ) $\mathrm{H} \alpha$ emission-line, with the most stringent constraints provided by our VLT GIRAFFE/ARGUS IFU spectra. Instead, these data show blue-asymmetric wings on the $\mathrm{H} \alpha$ and [ $\mathrm{N}$ II] line profiles indicative of a dusty outflow of ionized gas. While we cannot entirely exclude the possibility that an energetically significant Type 2 (obscured) AGN is present in some or all of the DCOs, we regard this possibility as unlikely. In particular, the DCOs are associated with galaxies having unusually weak $[\mathrm{SII}]$ and [OI] emission lines compared to normal star-forming galaxies. This is the opposite of what is seen in starburst-AGN composite systems (e.g., Kewley et al. 2006), but similar to what is seen in low redshift IR-warm starbursts (Kewley et al. 2001).

The continuum of the DCOs is dominated by the light of young stars: these are clearly strong highly compact starbursts. This is consistent with both the high gas pressures and emissionline profiles: both are commonly observed in starbursts and are the signposts of supernova-driven galactic winds (e.g., Lehnert \& Heckman 1996). We have considered two specific scenarios under which the starbursts associated with the DCOs might be unusual. They may be evolved short-duration starbursts in which the rate of photoionization is relatively low because few $\mathrm{O}$-stars are present. They may also be "leaky" starbursts in which a significant fraction of the ionizing radiation escapes (matter bounded conditions). The latter scenario seems less likely, since it is difficult to envision circumstances in which most of the ionizing radiation escapes the galaxy, but most of the nonionizing UV radiation is absorbed by dust and reprocessed in the mid/far IR.

We have compared the properties of the DCOs and other massive clumps to those of the nuclear star clusters typically seen in different kinds of nearby galaxies. The DCOs are highly massive and dense objects that do not appear to be related to single, young star clusters which are significantly less massive $\left(\leqslant 10^{7} M_{\odot}\right)$ and smaller $(\sim 10 \mathrm{pc})$ than the structures we see in the LBAs. However, the DCO structures are quite similar to predictions for the merger-driven starbursts that are believed to be the progenitors of the extra-light or central cusps seen generically in lower-mass $\left(\leqslant M_{*}\right)$ elliptical galaxies (Kormendy et al. 2009; Hopkins et al. 2009). This would be consistent with our optical images of the LBAs, most of which strongly suggest on-going mergers. However, at present it is unclear whether the DCO hosts could passively evolve to form a full-grown elliptical without additional merging. 
More generally, the properties of the LBAs are quite consistent with the idea that large Jeans instabilities in a gas-rich disk will lead to the formation of highly massive star-forming clumps. At high redshift, it is not yet clear what the main source of this gas is: it could be replenished continuously, or come in the form of discrete accretion events of small galaxies or clouds (or, most likely, a combination thereof). These processes have been proposed to explain the properties of high- $z$ star-forming galaxies (e.g., see Overzier et al. 2008; Basu-Zych et al. 2009; Elmegreen et al. 2009; Förster Schreiber et al. 2009; Law et al. 2007,2009 , and references therein). In any event, it is expected that the massive stellar clumps will aggregate at the center of a galaxy via dynamical friction to ultimately form a bulge and supermassive black hole (Elmegreen et al. 2009, and references therein). The LBAs offer us the opportunity of studying such processes in more detail, and compare samples across different redshifts.

We speculated about why we do not see clear evidence for the formation or rapid growth of a supermassive black hole in the DCOs (which appear to be the ideal black hole nurseries). One intriguing possibility is that these objects are simply too young for a black hole to be forming yet. Following Norman \& Scoville (1988) and Davies et al. (2007), the growth of a supermassive black hole inside a very massive compact star cluster may not commence until the cluster is old enough for the core-collapse supernovae phase to end (cluster age $>50 \mathrm{Myr}$ ). Subsequent to this, gas shed by evolved stars will be retained in the cluster's potential well rather than being blown out. This gas can then cool, flow inward, and be used to grow the black hole. If correct, we should be able to identify a population of the descendants of the young DCOs in a phase as powerful AGN. This scenario might also account for the rarity of a strong AGN in the LBG population (e.g., Steidel et al. 2002; Ouchi et al. 2008).

Follow-up UV spectroscopy using the HST Cosmic Origins Spectrograph has been allocated for selected targets in Cycle 17. The UV spectra will allow us to study many of the features discussed in this paper that are relevant to LBAs and to starbursts in general. These include the role of galactic outflows and low-luminosity AGNs, the escape of ionizing radiation, the strength of Ly $\alpha$ emission, stellar abundances and the initial mass function.

We are very grateful to Frederic Bournaud, Rychard Bouwens, Jarle Brinchmann, Bruce Elmegreen, Guinevere Kauffmann, Lisa Kewley, Isa Oliveira, Francesco Shankar, and the anonymous referee for useful suggestions and discussions. We thank Brent Groves for generating the optically thin models referred to in Section 4. We thank Anne Pellerin for her help with the WFPC2 reductions. We thank the support staff at ESO Paranal for their assistance with the FLAMES observations.

\section{REFERENCES}

Allen, M. G., Groves, B. A., Dopita, M. A., Sutherland, R. S., \& Kewley, L. J. 2008, ApJS, 178, 20

Arribas, S., Colina, L., Monreal Ibero, A., Alfonso, J., García-Marín, M., \& Alonso-Herrero, A. 2008, A\&A, 479, 687

Baker, A. J., Lutz, D., Genzel, R., Tacconi, L. J., \& Lehnert, M. D. 2001, A\&A, 372, L37

Baldwin, J. A., Phillips, M. M., \& Terlevich, R. 1981, PASP, 93, 5

Bastian, N. 2008, MNRAS, 390, 759

Basu-Zych, A. R., et al. 2007, ApJS, 173, 457

Basu-Zych, A., et al. 2009, ApJ, 699, L118

Beckwith, S. V. W., et al. 2006, AJ, 132, 1729
Bekki, K. 2004, PASA, 21, 167

Binette, L., Wilson, A. S., \& Storchi-Bergmann, T. 1996, A\&A, 312, 365

Binney, J., \& Tremaine, S. 2008, Galactic Dynamics (2nd ed.; Princeton, NJ: Princeton Univ. Press)

Böker, T., Sarzi, M., McLaughlin, D. E., van der Marel, R. P., Rix, H.-W., Ho, L. C., \& Shields, J. C. 2004, AJ, 127, 105

Bournaud, F., Elmegreen, B. G., \& Elmegreen, D. M. 2007, ApJ, 670, 237

Bournaud, F., et al. 2008, A\&A, 486, 741

Bournaud, F., \& Elmegreen, B. G. 2009, ApJ, 694, L158

Bouwens, R. J., Illingworth, G. D., Blakeslee, J. P., Broadhurst, T. J., \& Franx, M. 2004, ApJ, 611, L1

Bouwens, R. J., et al. 2009, ApJ, in press (arXiv:0909.4074)

Brinchmann, J., Pettini, M., \& Charlot, S. 2008a, MNRAS, 385, 769

Brinchmann, J., Kunth, D., \& Durret, F. 2008b, A\&A, 485, 657

Bruzual, G., \& Charlot, S. 2003, MNRAS, 344, 1000

Buat, V., Marcillac, D., Burgarella, D., Le Floc'h, E., Takeuchi, T. T., IglesiasParàmo, J., \& Xu, C. K. 2007, A\&A, 469, 19

Burgarella, D., Le Floc'h, E., Takeuchi, T. T., Huang, J. S., Buat, V., Rieke, G. H., \& Tyler, K. D. 2007, MNRAS, 380, 986

Burgarella, D., Buat, V., Takeuchi, T. T., Wada, T., \& Pearson, C. 2009, PASJ, 61,177

Calzetti, D. 2001, PASP, 113, 1449

Calzetti, D., et al. 2007, ApJ, 666, 870

Calzetti, D., Sheth, K., Churchwell, E., \& Jackson, J. 2009, in The Evolving ISM in the Milky Way and Nearby Galaxies, Fourth Spitzer Science Center Conf., ed. K. Sheth et al. (Pasadena, CA: Caltech), http://web.ipac. caltech.edu/staff/ismevol/proceedings/calzettid_v1.pdf

Caputi, K. I., et al. 2007, ApJ, 660, 97

Carollo, C. M., Stiavelli, M., de Zeeuw, P. T., \& Mack, J. 1997, AJ, 114, 2366

Castellanos, M., Díaz, Á. I., \& Tenorio-Tagle, G. 2002, ApJ, 565, L79

Chevalier, R. A., \& Clegg, A. W. 1985, Nature, 317, 44

Côté, P., et al. 2006, ApJS, 165, 57

Cowie, L. L., Hu, E. M., \& Songaila, A. 1995, AJ, 110, 1576

Crane, P., et al. 1993, AJ, 106, 1371

Daddi, E., et al. 2009, ApJ, 694, 1517

Dasyra, K. M., et al. 2006a, ApJ, 638, 745

Dasyra, K. M., et al. 2006b, ApJ, 651, 835

Davies, R. I., Sánchez, F. M., Genzel, R., Tacconi, L. J., Hicks, E. K. S., Friedrich, S., \& Sternberg, A. 2007, ApJ, 671, 1388

Dopita, M. A., \& Sutherland, R. S. 1995, ApJ, 455, 468

Elmegreen, D. M., Elmegreen, B. G., Rubin, D. S., \& Schaffer, M. A. 2005, ApJ, 631,85

Elmegreen, B. G., Bournaud, F., \& Elmegreen, D. M. 2008a, ApJ, 684, 829

Elmegreen, B. G., Bournaud, F., \& Elmegreen, D. M. 2008b, ApJ, 688, 67

Elmegreen, B. G., Elmegreen, D. M., Fernandez, M. X., \& Lemonias, J. J. 2009, ApJ, 692, 12

Eminian, C., Kauffmann, G., Charlot, S., Wild, V., Bruzual, G., Rettura, A., \& Loveday, J. 2008, MNRAS, 384, 930

Erb, D. K., Shapley, A. E., Pettini, M., Steidel, C. C., Reddy, N. A., \& Adelberger, K. L. 2006a, ApJ, 644, 813

Erb, D. K., Steidel, C. C., Shapley, A. E., Pettini, M., Reddy, N. A., \& Adelberger, K. L. 2006b, ApJ, 647, 128

Ferguson, H. C., et al. 2004, ApJ, 600, L107

Ferrarese, L., et al. 2006, ApJ, 644, L21

Förster Schreiber, N. M., et al. 2009, ApJ, submitted (arXiv:0903.1872)

Franx, M., Illingworth, G. D., Kelson, D. D., van Dokkum, P. G., \& Tran, K.-V. 1997, ApJ, 486, L75

Geha, M., Guhathakurta, P., \& van der Marel, R. P. 2002, AJ, 124, 3073

Genzel, R., Tacconi, L. J., Rigopoulou, D., Lutz, D., \& Tecza, M. 2001, ApJ, 563,527

Giammanco, C., Beckman, J. E., \& Cedrés, B. 2005, A\&A, 438, 599

Giavalisco, M. 2002, ARA\&A, 40, 579

Goldader, J. D., Meurer, G., Heckman, T. M., Seibert, M., Sanders, D. B., Calzetti, D., \& Steidel, C. C. 2002, ApJ, 568, 651

Graham, A. W. 2004, ApJ, 613, L33

Hayashi, M., et al. 2009, ApJ, 691, 140

Heckman, T. M., Armus, L., \& Miley, G. K. 1990, ApJS, 74, 833

Heckman, T. M., Miley, G. K., van Breugel, W. J. M., \& Butcher, H. R. 1981, ApJ, 247, 403

Heckman, T. M., Robert, C., Leitherer, C., Garnett, D. R., \& van der Rydt, F. 1998, ApJ, 503, 646

Heckman, T. M., et al. 2005, ApJ, 619, L35

Hoopes, C., et al. 2007, ApJS, 173, 441

Hopkins, A. M., Schulte-Ladbeck, R. E., \& Drozdovsky, I. O. 2002, AJ, 124, 862

Hopkins, P. F., Cox, T. J., Dutta, S. N., Hernquist, L., Kormendy, J., \& Lauer, T. R. 2009, ApJS, 181, 135 
Hopkins, P. F., Hernquist, L., Cox, T. J., Dutta, S. N., \& Rothberg, B. 2008, ApJ, 679,156

Huang, J.-S., et al. 2005, ApJ, 634, 137

Hughes, D. H., et al. 1998, Nature, 394, 241

Immeli, A., Samland, M., Westera, P., \& Gerhard, O. 2004, ApJ, 611, 20

Iwata, I., et al. 2009, ApJ, 692, 1287

Johnson, B. D., et al. 2007, ApJS, 173, 392

Kauffmann, G., \& Heckman, T. M. 2009, MNRAS, 397, 135

Kauffmann, G., et al. 2003, MNRAS, 346, 1055

Kewley, L. J., Groves, B., Kauffmann, G., \& Heckman, T. 2006, MNRAS, 372 , 961

Kewley, L. J., Heisler, C. A., Dopita, M. A., \& Lumsden, S. 2001, ApJS, 132, 37

Kissler-Patig, M., Jordán, A., \& Bastian, N. 2006, A\&A, 448, 1031

Kriek, M., et al. 2007, ApJ, 669, 776

Koekemoer, A. M., Fruchter, A. S., Hook, R. N., \& Hack, W. 2002, in The 2002 HST Calibration Workshop: Hubble after the Installation of the ACS and the NICMOS Cooling System, Proc. Workshop held at the Space Telescope Science Institute, Baltimore, MD, 2002, October 17 and 18, ed. S. Arribas, A. Koekemoer, \& B. Whitmore (Baltimore, MD: STScI), 337

Kong, X., Charlot, S., Brinchmann, J., \& Fall, S. M. 2004, MNRAS, 349, 769

Kormendy, J., Fisher, D. B., Cornell, M. E., \& Bender, R. 2009, ApJS, 182, 216

Krist, J. 1995, in ASP Conf. Ser. 77, Astronomical Data Analysis Software and Systems IV, ed. R. A. Shaw, H. E. Payne, \& J. J. E. Hayes (San Francisco, CA: ASP), 349

Kroupa, P. 2008, in ASP Conf. Ser. 390, Pathways Through an Eclectic Universe, ed. J. H. Knapen, T. J. Mahoney, \& A. Vazdekis (San Francisco, CA: ASP), 3

Lambas, D. G., Tissera, P. B., Alonso, M. S., \& Coldwell, G. 2003, MNRAS, 346, 1189

Larsen, S. S. 2002, AJ, 124, 1393

Larson, R. B., \& Tinsley, B. M. 1978, ApJ, 219, 46

Lauer, T. R., et al. 1995, AJ, 110, 2622

Lauer, T. R., et al. 2007, ApJ, 664, 226

Law, D. R., Steidel, C. C., Erb, D. K., Larkin, J. E., Pettini, M., Shapley, A. E., \& Wright, S. A. 2007, ApJ, 669, 929

Law, D. R., Steidel, C. C., Erb, D. K., Larkin, J. E., Pettini, M., Shapley, A. E., \& Wright, S. A. 2009, ApJ, 697, 2057

Lehnert, M. D., \& Heckman, T. M. 1996, ApJ, 462, 651

Lehnert, M. D., Nesvadba, N. P. H., Le Tiran, L., Di Matteo, P., van Driel, W., Douglas, L. S., Chemin, L., \& Bournaud, F. 2009, ApJ, 699, 1660

Leitherer, C., \& Heckman, T. M. 1995, ApJS, 96, 9

Li, C., Kauffmann, G., Heckman, T. M., Jing, Y. P., \& White, S. D. M. 2008, MNRAS, 385, 1903

Li, Y., Haiman, Z., \& Mac Low, M.-M. 2007, ApJ, 663, 61

Liu, X., Shapley, A. E., Coil, A. L., Brinchmann, J., \& Ma, C.-P. 2008, ApJ, 678,758

Lotz, J. M., Madau, P., Giavalisco, M., Primack, J., \& Ferguson, H. C. 2006, ApJ, 636, 592

Magorrian, J., et al. 1998, AJ, 115, 2285

Maraston, C., Bastian, N., Saglia, R. P., Kissler-Patig, M., Schweizer, F., \& Goudfrooij, P. 2004, A\&A, 416, 467

Marconi, A., \& Hunt, L. K. 2003, ApJ, 589, L21

Meurer, G. R., Heckman, T. M., \& Calzetti, D. 1999, ApJ, 521, 64

Meurer, G. R., Heckman, T. M., Leitherer, C., Kinney, A., Robert, C., \& Garnett, D. R. 1995, AJ, 110, 2665

Meurer, G. R., Heckman, T. M., Lehnert, M. D., Leitherer, C., \& Lowenthal, J. 1997, AJ, 114, 54

Mieske, S., et al. 2004, AJ, 128, 1529
Nikolic, B., Cullen, H., \& Alexander, P. 2004, MNRAS, 355, 874

Noguchi, M. 1999, ApJ, 514, 77

Norman, C., \& Scoville, N. 1988, ApJ, 332, 124

Osterbrock, D. E., \& Ferland, G. J. 2006, in Astrophysics of Gaseous Nebulae and Active Galactic Nuclei, ed. D. E. Osterbrock \& G. J. Ferland (2nd ed.; Sausalito, CA: Univ. Science Books)

Östlin, G., Hayes, M., Kunth, D., Mas-Hesse, J. M., Leitherer, C., Petrosian, A., \& Atek, H. 2009, AJ, 138, 923

Ouchi, M., et al. 2008, ApJS, 176, 301

Overzier, R. A., et al. 2008, ApJ, 677, 37

Owers, M., Blake, C., Couch, W., Pracy, M., \& Bekki, K. 2007, MNRAS, 381, 494

Papovich, C., Dickinson, M., \& Ferguson, H. C. 2001, ApJ, 559, 620

Papovich, C., Dickinson, M., \& Ferguson, H. C. 2006, ApJ, 640, 92

Pettini, M., \& Pagel, B. E. J. 2004, MNRAS, 348, L59

Reddy, N. A., \& Steidel, C. C. 2009, ApJ, 692, 778

Reddy, N. A., et al. 2006, ApJ, 644, 792

Rieke, G. H., Alonso-Herrero, A., Weiner, B. J., Pérez-González, P. G., Blaylock, M., Donley, J. L., \& Marcillac, D. 2009, ApJ, 692, 556

Rossa, J., van der Marel, R. P., Böker, T., Gerssen, J., Ho, L. C., Rix, H.-W., Shields, J. C., \& Walcher, C.-J. 2006, AJ, 132, 1074

Salim, S., et al. 2007, ApJS, 173, 267

Salpeter, E. E. 1955, ApJ, 121, 161

Sanders, D. B., \& Mirabel, I. F. 1996, ARA\&A, 34, 749

Seibert, M., et al. 2005, ApJ, 619, L55

Seth, A., Agüeros, M., Lee, D., \& Basu-Zych, A. 2008, ApJ, 678, 116

Shankar, F., \& Bernardi, M. 2009, MNRAS, 396, L76

Shapiro, K. L., et al. 2009, ApJ, 701, 955

Shapley, A. E., Steidel, C. C., Adelberger, K. L., Dickinson, M., Giavalisco, M., \& Pettini, M. 2001, ApJ, 562, 95

Shapley, A. E., Coil, A. L., Ma, C.-P., \& Bundy, K. 2005, ApJ, 635, 1006

Shapley, A. E., Steidel, C. C., Pettini, M., Adelberger, K. L., \& Erb, D. K. 2006, ApJ, 651, 688

Siana, B., Teplitz, H. I., Chary, R.-R., Colbert, J., \& Frayer, D. T. 2008, ApJ, 689,59

Spinoglio, L., Andreani, P., \& Malkan, M. A. 2002, ApJ, 572, 105

Stark, D. P., Swinbank, A. M., Ellis, R. S., Dye, S., Smail, I. R., \& Richard, J 2008, Nature, 455, 775

Stark, D. P., Ellis, R. S., Bunker, A., Bundy, K., Targett, T., Benson, A., \& Lacy, M. 2009, ApJ, 697, 1493

Steidel, C. C., Adelberger, K. L., Giavalisco, M., Dickinson, M., \& Pettini, M. 1999, ApJ, 519, 1

Steidel, C. C., Hunt, M. P., Shapley, A. E., Adelberger, K. L., Pettini, M., Dickinson, M., \& Giavalisco, M. 2002, ApJ, 576, 653

Tacconi, L. J., Genzel, R., Lutz, D., Rigopoulou, D., Baker, A. J., Iserlohe, C., \& Tecza, M. 2002, ApJ, 580, 73

Telles, E., \& Terlevich, R. 1997, MNRAS, 286, 183

Teplitz, H. I., et al. 2000, ApJ, 533, L65

Tremonti, C. A., et al. 2004, ApJ, 613, 898

Treyer, M., et al. 2007, ApJS, 173, 256

Vázquez, G. A., \& Leitherer, C. 2005, ApJ, 621, 695

Veilleux, S., \& Osterbrock, D. E. 1987, ApJS, 63, 295

Walcher, C. J., et al. 2005, ApJ, 618, 237

Wehner, E. H., \& Harris, W. E. 2006, ApJ, 644, L17

Weidner, C., Kroupa, P., \& Larsen, S. S. 2004, MNRAS, 350, 1503

Whitmore, B. C., Chandar, R., \& Fall, S. M. 2007, AJ, 133, 1067

Whittle, M., Pedlar, A., Meurs, E. J. A., Unger, S. W., Axon, D. J., \& Ward, M. J. 1988, ApJ, 326, 125

Woods, D. F., Geller, M. J., \& Barton, E. J. 2006, AJ, 132, 197 